\title{
ESTIMATION AND FILTERING OF FRACTIONAL GENERALISED RANDOM FIELDS
}

\author{
J. M. ANGULO, M. D. RUIZ-MEDINA and V. V. ANH
}

(Received 25 June 1998; revised 05 April 2000)

Communicated by V. Stefanov

\begin{abstract}
This paper considers the estimation and filtering of fractional random fields, of which fractional Brownian motion and fractional Riesz-Bessel motion are important special cases. A least-squares solution to the problem is derived by using the duality theory and covariance factorisation of fractional generalised random fields. The minimum fractional duality order of the information random field leads to the most general class of solutions corresponding to the largest function space where the output random field can be approximated. The second-order properties that define the class of random fields for which the least-squares linear estimation problem is solved in a weak-sense are also investigated in terms of the covariance spectrum of the information random field.
\end{abstract}

2000 Mathematics subject classification: primary 60G60; secondary 60G20.

Keywords and phrases: Wiener filtering;estimation of random fields; fractional generalised random fields.

\section{Introduction}

Let the second-order ordinary random field $\left\{\mathscr{Y}(\mathbf{z}): \mathbf{z} \in T \subseteq \mathbb{R}^{d}\right\}$ be the output of a linear system, defined in terms of the linear operator $A$, with random input $\mathscr{S}$. That is,

$$
\mathscr{Y}(\mathbf{z})=A \mathscr{S}(\mathbf{z}), \quad \forall \mathbf{z} \in T \subseteq \mathbb{R}^{d} .
$$

The ordinary estimation problem considered here consists of calculating the leastsquares linear estimate of $\mathscr{Y}(\mathbf{z})$, for each $\mathbf{z} \in T$, from the information provided by the following observation model:

$$
\mathscr{X}(\mathbf{x})=\mathscr{S}(\mathbf{x})+\mathscr{N}(\mathbf{x}), \quad \mathbf{x} \in T_{\mathscr{X}} \subseteq T,
$$

This work was partially supported by the Programa Sectorial de Promoción general del Conocimiento (Project PB96-1440) of the Dirección General de Enseñanza Superior, Ministerio de Educación y Cultura, Spain, and the Australian Research Council grant A89601825.

(C) 2000 Australian Mathematical Society 0263-6115/2000\$A2.00+0.00 
where $\mathscr{N}$ is a zero-mean additive noise. The covariance function $B_{\mathscr{X}}(\mathbf{x}, \mathbf{y})=$ $E[\mathscr{X}(\mathbf{x}) \overline{\mathscr{X}}(\mathbf{y})]$, for $\mathbf{x}, \mathbf{y} \in T_{\mathscr{X}}$, as well as the cross-covariance function $B_{\mathscr{X} \mathscr{Y}}(\mathbf{x}, \mathbf{z})=$ $f(\mathbf{x}, \mathbf{z})=E[\mathscr{X}(\mathbf{x}) \overline{\mathscr{Y}}(\mathbf{z})]$, for $\mathbf{x} \in T_{\mathscr{X}}$ and $\mathbf{z} \in T$, are assumed to be known. From the Orthogonal Projection Theorem, for each $\mathrm{z} \in T$, the least-squares linear estimate $K \mathscr{X}(\mathbf{z})$ of $\mathscr{Y}(\mathbf{z})$, based on the information provided by $\mathscr{X}$ in (1.2), is defined as

$$
\widehat{\mathscr{Y}(\mathbf{z})}=K \mathscr{X}(\mathbf{z}):=\int_{T_{\mathscr{x}}} k(\mathbf{z}, \mathbf{x}) \mathscr{X}(\mathbf{x}) d \mathbf{x},
$$

where $k(\mathbf{z}, \cdot)$ is the solution to the following system:

$$
\begin{aligned}
& E[(\mathscr{Y}(\mathbf{z})-K \mathscr{X}(\mathbf{z})) \mathscr{X}(\mathbf{x})]=0 \\
\Longleftrightarrow \quad & B_{\mathscr{Y} \mathscr{X}}(\mathbf{z}, \mathbf{x})=K B_{\mathscr{X}}(\mathbf{z}, \mathbf{x})=\int_{T_{\mathscr{X}}} k(\mathbf{z}, \mathbf{y}) B_{\mathscr{X}}(\mathbf{y}, \mathbf{x}) d \mathbf{y}, \quad \forall \mathbf{x} \in T_{\mathscr{X}} .
\end{aligned}
$$

In applications $A$ is known, since it defines the equation of the system studied. Hence, the cross-covariance function between $\mathscr{Y}$ and $\mathscr{X}$ can be calculated in terms of the cross-covariance function between the signal $\mathscr{S}$ and the information random field $\mathscr{X}$. Furthermore, in the case where $A$ is the identity operator, the above estimation problem is a filtering problem, and in the case where $A$ is a shift operator, we have an extrapolation problem.

For each $\mathbf{z} \in T$, the solution $k(\mathbf{z}, \cdot)$ to (1.4) usually is not in $L^{2}\left(T_{\mathscr{X}}\right)$ and therefore, regularisation methods must be applied to solve such an equation.

A solution of the estimation problem for a general class of random fields $\mathscr{X}$ whose covariance operators $R_{\mathscr{X}}$ are rational functions of positive polynomials of a self-adjoint elliptic differential operator $\mathscr{L}$ of integer order on $L^{2}\left(\mathbb{B}^{d}\right)$ was detailed in Ramm [20] (see also Ramm [21]). A covariance operator $R_{\mathscr{X}}$ in this class is defined as

$$
\begin{aligned}
R_{\mathscr{X}}(f)(\mathbf{x}) & =P(\mathscr{L}) Q^{-1}(\mathscr{L})(f)(\mathbf{x})=\int_{T_{\mathscr{X}}} B_{\mathscr{X}}(\mathbf{x}, \mathbf{y}) f(\mathbf{y}) d \mathbf{y} \\
& =\int_{T_{\mathscr{X}}}\left[\int_{\Lambda} P(\lambda) Q^{-1}(\lambda) \Phi(\mathbf{x}, \mathbf{y}, \lambda) d \rho(\lambda)\right] f(\mathbf{y}) d \mathbf{y},
\end{aligned}
$$

for every function $f$ in the domain of $R_{\mathscr{X}}$. Here $\Lambda, d \rho, \Phi(\cdot, \cdot, \cdot)$ are respectively the spectrum, the spectral measure and the spectral kernel of a self-adjoint elliptic differential operator $\mathscr{L}$ on $L^{2}\left(\mathbb{B}^{d}\right)$ of integer order $s$, and $P(\cdot)$ and $Q(\cdot)$ are positive polynomials of degrees $p$ and $q$, respectively. The polynomials $P$ and $Q$ are assumed to satisfy the conditions

$$
0<c_{1} \leq P(\lambda)\left(1+|\lambda|^{2}\right)^{-p / 2} \leq c_{2}, \quad 0<c_{3} \leq Q(\lambda)\left(1+|\lambda|^{2}\right)^{-q / 2} \leq c_{4} .
$$

This class of non-homogeneous random fields covers, as special cases, many wellknown classes in the theory of linear filtering. 
Recent advances in stochastic analysis have added a new dimension to the problem, namely, filtering of fractal processes. These processes arise in many applications in turbulence, geostatistics, hydrology, image compression, biological systems, financial modelling, etc. (see, for example, Farge et al. [13], Barnsley and Hurd [7], Beran [8], Peters [19], Frisch [14], Barabasi and Stanley [6], Innacone and Khokha [16], Falconer [12], Anh and Heyde [3]). Two fundamental examples of fractal processes are fractional Brownian motion ( $\mathrm{fBm})$, which is characterised by a spectral density of the form

$$
f(\lambda)=\frac{c}{|\lambda|^{2 \gamma}} \frac{|\lambda|^{2}}{1+|\lambda|^{2}}, \quad c>0,1 / 2<\gamma<3 / 2, \lambda \in \mathbb{R}^{d},
$$

and fractional Riesz-Bessel motion (fRBm), which is characterised by a spectral density of the form

$$
f(\lambda)=\frac{c}{|\lambda|^{2 \gamma}} \frac{1}{\left(1+|\lambda|^{2}\right)^{\alpha}} \frac{|\lambda|^{2}}{1+|\lambda|^{2}}, \quad c>0,1 / 2<\gamma<3 / 2, \alpha>0, \lambda \in \mathbb{R}^{d}
$$

(see Mandelbrot and Van Ness [18], Anh et al. [4], Anh et al. [1]). The component $|\lambda|^{-2 \gamma}$ with $1<\gamma<3 / 2$ models the long-range dependence of the process, while the component $\left(1+|\lambda|^{2}\right)^{-\alpha}$ with $\alpha>0$ models its second-order intermittency (Anh et $a l$. [2]). Noting that the exponents $\alpha$ and $\gamma$ in (1.4) and (1.5) are real numbers, it is seen that the filtering problem for fractals must be solved in the framework of Sobolev spaces of fractional order.

In this paper, we develop a theory for estimation and filtering of a general class of fractional generalised random fields (FGRF), of which $\mathrm{fBm}$ and $\mathrm{fRBm}$ are important examples. The needed tools including the duality theory and covariance factorisation of FGRFs have been obtained in Ruiz-Medina et al. [23].

Apart from offering a suitable framework for extending existing results to fractals, the consideration of FGRFs in an estimation theory has two important advantages: The second-order regularity properties of the random fields involved in the estimation problem refer to a continuous scale given by the orders of the fractional Sobolev spaces where an FGRF can be defined. The other advantage is that the duality condition relative to a fractional Sobolev space (see Ruiz-Medina et al. [23] for the fractional case; Anh et al. [5] for the integer case) allows us to specify the function space where the weak-sense solution to the estimation problem is found and where the observation of the information random field must be considered.

We first consider the fractional weak-sense interpretation of the initial equation which represents the relationship between the input and output random fields. The observations provided by the information random field are collected by means of test functions in a fractional Sobolev space according to its second-order regularity properties. The weak-sense least-squares linear estimation of the output random field 
is then calculated as the solution with minimum fractional singularity order to the fractional generalised version of the Wiener-Hopf equation in the reproducing kernel Hilbert space (RKHS) of the fractional dual of the information random field. The class of random fields for which the estimation problem is solved in a weak sense is characterised through the properties of the spectrum of the covariance operator of the information random field. An important case is the class of random fields whose covariance operator is a rational function of positive fractional polynomials of a self-adjoint elliptic differential operator of fractional order on $L^{2}(T), T \subseteq \mathbb{R}^{d}$. This special case provides an extension of Ramm's estimation theory.

\section{Preliminaries}

In this section we introduce some definitions and results from the theory of fractional generalised random fields developed in Ruiz-Medina et al. [23]. The second-order regularity properties of a fractional generalised random field are studied in terms of the regularity properties of the functions belonging to an appropriate fractional Sobolev space via the RKHS theory. We first consider the basic definitions of function spaces with fractional Sobolev norms.

Let $C_{0}^{\infty}\left(\mathbb{R}^{d}\right)$ be the space of infinitely differentiable functions with compact support contained in $\mathbb{R}^{d}$, and let $\mathscr{S}\left(\mathbb{R}^{d}\right)$ be the space of $C^{\infty}$-functions with rapid decay at infinity. The duals of these spaces are respectively known as the space of distributions, $\left[C_{0}^{\infty}\left(\mathbb{R}^{d}\right)\right]^{\prime}$, and the space of tempered distributions, $\mathscr{S}^{\prime}\left(\mathbb{R}^{d}\right)$. The relationship between them is given by the following inclusions:

$$
C_{0}^{\infty}\left(\mathbb{R}^{d}\right) \subseteq \mathscr{S}\left(\mathbb{R}^{d}\right) \subseteq \mathscr{S}^{\prime}\left(\mathbb{R}^{d}\right) \subseteq\left[C_{0}^{\infty}\left(\mathbb{R}^{d}\right)\right]^{\prime} .
$$

Similarly, $C_{0}^{\infty}(T)$, with $T \subseteq \mathbb{R}^{d}$, represents the space of infinitely differentiable functions with compact support contained in $T$, and $\left[C_{0}^{\infty}(T)\right]^{\prime}$ the space of distributions on $T$. In relation to these spaces the following concepts are fundamental.

DEFINITION 2.1 (Dautray and Lions [10, pages 474-475]). Restriction of a distribution to an open set. Consider two open sets $T$ and $T^{\prime}$ of $R^{d}$, with $T \subset T^{\prime}$, and let $F$ be a distribution on $T^{\prime}$. The restriction $F_{T}$ of $F$ to $T$ is defined as follows: For all $\varphi \in C_{0}^{\infty}(T)$,

$$
\left\langle F_{T}, \varphi\right\rangle=\langle F, \tilde{\varphi}\rangle,
$$

where $\tilde{\varphi}$ is the extension by 0 of $\varphi$ to $T^{\prime}$.

Support of a distribution. Let $F$ be a distribution on $T$, with $T \subseteq R^{d}$. The support of $F$, denoted by supp $F$, is the complement of the largest open set $S$ of $T$ such that the restriction of $F$ to $S$ is null. 
The continuous scale of fractional Sobolev spaces is introduced in terms of Bessel potentials $\left\{\mathscr{I}_{-s}=(I-\Delta)^{-s / 2}: s \in \mathbb{R}\right\}$, with $I$ representing the identity operator and $-\Delta$ being the negative Laplacian operator (see Stein [25]). The order $s$ of such potentials provides information about the regularity properties of the functions in these spaces through the Fourier transform.

DEFINITION 2.2. For $s \in R, H^{s}\left(R^{d}\right)$ is the space of tempered distributions $u$ such that

$$
\left(1+|\lambda|^{2}\right)^{s / 2} \hat{u}(\lambda) \in L^{2}\left(\mathbb{R}^{d}\right), \quad \lambda \in \mathbb{R}^{d},
$$

where ${ }^{\wedge}$ stands for the Fourier transform. In this space the following inner product is considered:

with associated norm

$$
(u, v)_{s}=\int_{\mathbb{R}^{d}}\left(1+|\lambda|^{2}\right)^{s} \hat{u}(\lambda) \hat{v}(\lambda) d \lambda,
$$

$$
\|u\|_{s}=\left(\int_{\mathbb{R}^{d}}\left(1+|\lambda|^{2}\right)^{s}|\hat{u}(\lambda)|^{2} d \lambda\right)^{1 / 2} .
$$

REMARK 2.1. For $s_{1} \geq s_{2} \geq 0$, the following inclusions hold:

$$
\begin{aligned}
C_{0}^{\infty}\left(\mathbb{R}^{d}\right) & \subseteq \mathscr{S}\left(\mathbb{R}^{d}\right) \subseteq H^{s_{1}}\left(\mathbb{R}^{d}\right) \subseteq H^{s_{2}}\left(\mathbb{R}^{d}\right) \subseteq H^{0}\left(\mathbb{R}^{d}\right) \\
& \equiv L_{2}\left(\mathbb{R}^{d}\right) \subseteq H^{-s_{2}}\left(\mathbb{R}^{d}\right) \subseteq H^{-s_{1}}\left(\mathbb{R}^{d}\right) \subseteq \mathscr{S}^{\prime}\left(\mathbb{R}^{d}\right) \subseteq\left[C_{0}^{\infty}\left(\mathbb{R}^{d}\right)\right]^{\prime} .
\end{aligned}
$$

For $s \in \mathbb{R}, H^{s}\left(\mathbb{R}^{d}\right)$ and $H^{-s}\left(\mathbb{R}^{d}\right)$ are dual Hilbert spaces.

From the above definitions the following fractional Sobolev spaces on a bounded $C^{\infty}$-domain $T \subset \mathbb{R}^{d}$ are introduced:

DEFINITION 2.3 (Triebel [26, page 310]). For $s \in R, H^{s}(T)$ is the restriction to $T$ of $H^{s}\left(R^{d}\right)$. That is,

$$
H^{s}(T)=\left\{f \in\left[C_{0}^{\infty}(T)\right]^{\prime}: \exists F \in H^{s}\left(\mathbb{R}^{d}\right) \text { such that } f=F_{T}\right\},
$$

where $F_{T}$ denotes the restriction of $F$ to $T$. With the quotient norm

$$
\|f\|_{H^{s}(T)}=\inf _{F ; F_{r}=f}\|F\|_{H^{s}\left(\mathbb{R}^{d}\right)},
$$

$H^{s}(T)$ is a Hilbert space.

The space $\bar{H}^{s}(T)$ represents the set of functions in $H^{s}\left(R^{d}\right)$ with support contained in $T$. That is,

$$
\bar{H}^{s}(T)=\left\{u \in H^{s}\left(\mathbb{R}^{d}\right): \operatorname{supp} u \subseteq \bar{T}\right\}={\overline{C_{0}^{\infty}(T)^{\|}}}^{\|\|_{H^{s}\left(\mathbb{R}^{d}\right)}} .
$$


For $s \in \mathbb{R}$, the spaces $\bar{H}^{s}(T)$ and $H^{-s}(T)$ are dual Hilbert spaces. Furthermore,

$$
H^{s}(T)=H^{s}\left(\mathbb{R}^{d}\right) / \bar{H}^{s}\left(\mathbb{R}^{d} \backslash T\right)
$$

(see Triebel [26, page 317]).

REMARK 2.2. Note that the dual space of $\bar{H}^{s}(T)$, that is, the space $H^{-s}(T)$, for $s \in \mathbb{R}_{+}$, is algebraically and topologically equivalent to the space $\mathscr{I}_{2 s}\left(\bar{H}^{s}(T)\right)=$ $(I-\Delta)^{s}\left(\bar{H}^{s}(T)\right)$, with operator $\mathscr{I}_{2 s}$ defined on domain $T$. The dual space of $H^{-s}(T)$, that is, $\bar{H}^{s}(T), s \in \mathbb{R}_{+}$, is also obtained as $\mathscr{I}_{-2 s}\left(H^{-s}(T)\right)=(I-\Delta)^{-s}\left(H^{-s}(T)\right)$.

From Sobolev's Embedding Theorem (see, for example, Dautray and Lions [10]), the functions in the Sobolev space $\bar{H}^{s}(T)$ with $[s]^{-}>d / 2$, where $[\cdot]^{-}$represents the integer part function, are continuous, and the dual space $H^{-s}(T)$ consists of distributions with compact support and order $[s-d / 2]^{-}$(see Schwartz [24, page 26]).

In the following development we denote by $U$ the space $C_{0}^{\infty}(T)$ with the topology defined as the intersection of the topologies associated with the Sobolev spaces of fractional order $\left\{\bar{H}^{\alpha}(T): \alpha \in \mathbb{Q}\right\}$. The space $U$ is a countably Hilbert space. We denote by $U_{\alpha}$ and $V_{\alpha}$ the dual fractional Sobolev spaces $\bar{H}^{\alpha}(T)$ and $H^{-\alpha}(T)$, with $\alpha \in \mathbb{Q}$, respectively (see, for example, Triebel [26]; Dautray and Lions [10]).

Let $\mathscr{L}^{2}(\Omega, \mathscr{A}, P)$ be the Hilbert space of real-valued zero-mean random variables defined on the basic probability space $(\Omega, \mathscr{A}, P)$ with finite second-order moments and with the inner product

$$
\langle X, Y\rangle_{\mathscr{L}^{2}(\Omega)}=E[X Y], \quad X, Y \in \mathscr{L}^{2}(\Omega, \mathscr{A}, P) .
$$

DEFINITION 2.4. For $\alpha \in Q$, a random function $X_{\alpha}(\cdot)$ from $U_{\alpha}$ into $L^{2}(\Omega, A, P)$ is said to be an $\alpha$-generalised random field ( $\alpha$-GRF) if it is linear and continuous in the mean-square sense with respect to the $U_{\alpha}$-topology.

For a continuous linear random function $X$ from $C_{0}^{\infty}(T)$ into $\mathscr{L}^{2}(\Omega, \mathscr{A}, P)$, that is, for a generalised random field (GRF) $X$ in the sense of Rozanov [22], there always exists a continuous extension to the space $U_{\alpha}$, for some $\alpha \in \mathbb{Q}$ (see Gel'fand and Vilenkin [15, page 74]). Therefore, jointly with the definition of a GRF $X$, a fractional $\alpha$-GRF $X_{\alpha}$ is considered, for some $\alpha \in \mathbb{Q}$. We refer to the minimum order for which such an extension exists as the minimum fractional singularity order (or the maximum fractional regularity order) of $X$.

REMARK 2.3. Note that the minimum fractional singularity order $\alpha$ of an ordinary random field $\mathscr{X}$ is non positive and provides information about the regularity order of its covariance function $B_{\mathscr{X}}$. Such an order determines the function space where $\mathscr{X}$ is mean-square integrable, or equivalently where its covariance operator $R_{\mathscr{X}}$ can 
be defined. Thus, if an ordinary random field $\mathscr{X}$ has minimum fractional singularity order $\alpha$, then

$$
\left(R_{\mathscr{X}}(f), f\right)=\int_{T} \int_{T} B_{\mathscr{X}}(\mathbf{z}, \mathbf{y}) f(\mathbf{y}) f(\mathbf{z}) d \mathbf{y} d \mathbf{z}<\infty, \quad \forall f \in \bar{H}^{\alpha}(T) .
$$

Since $\alpha \leq 0$, the function $f$ in (2.4) is a distribution and such an equation makes sense only when $B_{\mathscr{T}}$ belongs to the space of test functions $H^{-\alpha}(T)$ where the distributions of $\bar{H}^{\alpha}(T)$ are defined. Thus, the regularity order $-\alpha$ of the mentioned test functions compensates the singularity order $\alpha$ of the corresponding distributions. Moreover, in the case where $[-\alpha]^{-}>d / 2$, the covariance function $B_{\mathscr{X}}$ of $\mathscr{X}$ is continuous and, therefore, the ordinary random field $\mathscr{X}$ is continuous in the mean-square sense.

The second-order regularity properties of an $\alpha$-GRF $X_{\alpha}$ are studied in terms of the following Hilbert spaces: The Hilbert space $H\left(X_{\alpha}\right)$ defined as the closed span in the $\mathscr{L}^{2}(\Omega)$-topology of $\left\{X_{\alpha}(\varphi): \varphi \in U_{\alpha}\right\}$, and the RKHS $\mathscr{H}\left(X_{\alpha}\right)$, which is the closed span in the $\mathscr{L}^{2}(\Omega)$-topology of $\left\{B_{\alpha}(\phi, \cdot)=E\left[X_{\alpha}(\phi) X_{\alpha}(\cdot)\right]: \phi \in U_{\alpha}\right\}$. The inner product defined in the space $H\left(X_{\alpha}\right)$ is given by (2.3). Each function $u$ in the RKHS is defined as

$$
u(\phi)=E\left[X_{u} X_{\alpha}(\phi)\right], \quad \forall \phi \in U_{\alpha},
$$

for a certain $X_{u} \in H\left(X_{\alpha}\right)$. That is, we can establish an isometric isomorphism between the spaces $\mathscr{H}\left(X_{\alpha}\right)$ and $H\left(X_{\alpha}\right)$ associating to each function $u \in \mathscr{H}\left(X_{\alpha}\right)$ the random variable $X_{u} \in H\left(X_{\alpha}\right)$ defined by (2.5). Therefore, the inner product of two functions in the RKHS is given by the inner product in $\mathscr{L}^{2}(\Omega)$ of the random variables defining such functions according to (2.5).

The covariance function $B_{\alpha}$ of the $\alpha$-GRF $X_{\alpha}$ defines a continuous bilinear form on $\bar{H}^{\alpha}(T) \otimes \bar{H}^{\alpha}(T)$, since from Definition 2.4 the $\alpha$-GRF $X_{\alpha}$ is continuous in the meansquare sense. From the Kernel Theorem (Gel'fand and Vilenkin [15]), $B_{\alpha}$ admits the representation

$$
B_{\alpha}(\varphi, \phi)=\left\langle\left(R_{\alpha} \varphi\right)^{*}, \phi\right\rangle_{U_{\alpha}}, \quad \varphi, \phi \in U_{\alpha},
$$

where $*$ stands for the duality between Hilbert spaces (Riesz Representation Theorem), and $R_{\alpha}$ is the covariance operator of $X_{\alpha}$, that is, a symmetric positive continuous linear operator from $U_{\alpha}$ into $V_{\alpha}$.

DEFINITION 2.5. For $\alpha \in Q$, we say that the GRF

$$
\tilde{X}_{\alpha}: V_{\alpha} \rightarrow \mathscr{L}^{2}(\Omega, \mathscr{A}, P)
$$

is the dual relative to $U_{\alpha}$ (or $\alpha$-dual) of the $\alpha$-GRF

$$
X_{\alpha}: U_{\alpha} \rightarrow \mathscr{L}^{2}(\Omega, \mathscr{A}, P)
$$

if it satisfies: 
(i) $H\left(X_{\alpha}\right)=H\left(\tilde{X}_{\alpha}\right)$,

(ii) $\left\langle X_{\alpha}(\phi), \tilde{X}_{\alpha}(g)\right\rangle_{H\left(X_{\alpha}\right)}=\left\langle\phi, g^{*}\right\rangle_{U_{\alpha}}$, for $\phi \in U_{\alpha}$, and $g \in V_{\alpha}$, with $g^{*}$ being the dual element of $g$ with respect to the $U_{\alpha}$-topology.

Conversely, the dual of $\tilde{X}_{\alpha}$ relative to $V_{\alpha}$ is the $\alpha$-GRF $X_{\alpha}$.

REMARK 2.4. The $\alpha$-dual GRF $\tilde{X}_{\alpha}$ can be interpreted as the adjoint of the inverse of the $\alpha$-GRF $X_{\alpha}: U_{\alpha} \rightarrow \mathscr{L}^{2}(\Omega, \mathscr{A}, P)$. Therefore, as we show below, the covariance operators of both GRFs are isomorphisms between the fractional Sobolev spaces involved in their definitions. Indeed, the duality condition is equivalent to the existence of a bounded inverse $R_{\alpha}^{-1}$ from $V_{\alpha}$ onto $U_{\alpha}$ for the covariance operator $R_{\alpha}$. In the abstract representation we derive in Theorem 2.3, the $\alpha$-dual GRF defines the inverse of the linear filter (2.20) relating $X_{\alpha}$ with white noise.

Similarly, associated with the $\alpha$-dual GRF $\tilde{X}_{\alpha}$, we also consider the spaces $H\left(\tilde{X}_{\alpha}\right)$ and $\mathscr{H}\left(\tilde{X}_{\alpha}\right)$, defined as the closed spans in the $\mathscr{L}^{2}(\Omega)$-topology of the following sets:

$$
\left\{\tilde{X}_{\alpha}(f): f \in V_{\alpha}\right\} \quad \text { and } \quad\left\{\tilde{B}_{\alpha}(f, \cdot)=E\left[\tilde{X}_{\alpha}(f) \tilde{X}_{\alpha}(\cdot)\right]: f \in V_{\alpha}\right\},
$$

respectively. The spaces $H\left(X_{\alpha}\right)$ and $\mathscr{H}\left(X_{\alpha}\right)$, and correspondingly $H\left(\tilde{X}_{\alpha}\right)$ and $\mathscr{H}\left(\tilde{X}_{\alpha}\right)$, can be related by means of the isometric isomorphisms

$$
J: H\left(X_{\alpha}\right) \rightarrow \mathscr{H}\left(X_{\alpha}\right) \text { and } J^{\prime}: H\left(\tilde{X}_{\alpha}\right) \rightarrow \mathscr{H}\left(\tilde{X}_{\alpha}\right)
$$

respectively, which are defined as follows:

$$
\begin{array}{ll}
Y \rightarrow J Y, \quad \text { with } \quad(J Y)(\phi)=E Y X_{\alpha}(\phi), \quad \forall \phi \in U_{\alpha}, \\
Z \rightarrow J^{\prime} Z, \quad \text { with } \quad\left(J^{\prime} Z\right)(g)=E Z \tilde{X}_{\alpha}(g), \quad \forall g \in\left[U_{\alpha}\right]^{*} .
\end{array}
$$

As $\mathscr{H}\left(X_{\alpha}\right) \subseteq\left[U_{\alpha}\right]^{*}$ and $\mathscr{H}\left(\tilde{X}_{\alpha}\right) \subseteq U_{\alpha}$, the following operators can be considered:

$$
\begin{aligned}
& \mathscr{X}: \mathscr{H}\left(X_{\alpha}\right) \rightarrow\left[U_{\alpha}\right]^{*}, \quad \text { with } \quad g \rightarrow \mathscr{K} g=g, \\
& \mathscr{K}^{\prime}: \mathscr{H}\left(\tilde{X}_{\alpha}\right) \rightarrow U_{\alpha}, \quad \text { with } \quad \phi \rightarrow \mathscr{K}^{\prime} \phi=\phi .
\end{aligned}
$$

The composition of the operators $J$ and $\mathscr{K}^{\prime}$, and $J^{\prime}$ and $\mathscr{K}^{\prime}$, respectively, leads to the introduction of the operators $S_{\alpha}$ and $S_{\alpha}^{\prime}$, defined as

$$
\begin{aligned}
& S_{\alpha}:=\mathscr{K} J: H\left(X_{\alpha}\right) \rightarrow\left[U_{\alpha}\right]^{*}, \\
& S_{\alpha}^{\prime}:=\mathscr{K}^{\prime} J^{\prime}: H\left(\tilde{X}_{\alpha}\right) \rightarrow U_{\alpha} .
\end{aligned}
$$

The minimum fractional order $\tilde{\alpha}$ for which the dual GRF of $X_{\alpha}$ relative to $U_{\beta}$, with $\beta \geq \alpha$, exists is called the minimum fractional duality order of $X_{\alpha}$. This order determines the spaces $U_{\tilde{\alpha}},\left[U_{\tilde{\alpha}}\right]^{*}, H\left(X_{\tilde{\alpha}}\right), H\left(\tilde{X}_{\tilde{\alpha}}\right), \mathscr{H}\left(X_{\tilde{\alpha}}\right)$, and $\mathscr{H}\left(\tilde{X}_{\tilde{\alpha}}\right)$ in terms of which equations (2.10)-(2.14) are defined. In the following proposition, the bijectivity and bicontinuity of the operators $S_{\bar{\alpha}}$ and $S_{\tilde{\alpha}}^{\prime}$ are established. 
PROPOSITION 2.1. Let $X_{\alpha}$ be an $\alpha$-GRF. Assume the $\tilde{\alpha}$-GRF $\tilde{X}_{\alpha}$ exists. Then, the following assertions hold:

(i) The operators $S_{\tilde{\alpha}}^{\prime} X_{\tilde{\alpha}}$ and $X_{\tilde{\alpha}} S_{\tilde{\alpha}}^{\prime}$ are the identity operators on the spaces $U_{\tilde{\alpha}}$ and $H\left(X_{\tilde{\alpha}}\right)$, respectively. Conversely, the operators $S_{\tilde{\alpha}} \tilde{X}_{\tilde{\alpha}}$ and $\tilde{X}_{\tilde{\alpha}} S_{\tilde{\alpha}}$ are the identity operators on the spaces $\left[U_{\tilde{\alpha}}\right]^{*}$ and $H\left(\tilde{X}_{\tilde{\alpha}}\right)=H\left(X_{\tilde{\alpha}}\right)$, respectively.

(ii) The operators $S_{\tilde{\alpha}}$ and $S_{\tilde{\alpha}}^{\prime}$ are bicontinuous.

PROOF. (i) First, we prove that $\mathscr{H}\left(X_{\tilde{\alpha}}\right)=\left[U_{\tilde{\alpha}}\right]^{*}$ and $\mathscr{H}\left(\tilde{X}_{\tilde{\alpha}}\right)=U_{\tilde{\alpha}}$, as sets of functions. By definition, $\mathscr{H}\left(X_{\tilde{\alpha}}\right) \subseteq\left[U_{\tilde{\alpha}}\right]^{*}$. From the duality between $X_{\tilde{\alpha}}$ and $\tilde{X}_{\tilde{\alpha}}$ relative to $U_{\tilde{\alpha}}$, we have that, for each $g \in\left[U_{\tilde{\alpha}}\right]^{*}$,

$$
g(\varphi)=\left\langle g^{*}, \varphi\right\rangle_{U_{\tilde{\alpha}}}=\left\langle\tilde{X}_{\tilde{\alpha}}(g), X_{\tilde{\alpha}}(\varphi)\right\rangle_{H\left(X_{\tilde{\alpha}}\right)}=J\left[\tilde{X}_{\tilde{\alpha}}(g)\right](\varphi), \quad \forall \varphi \in U_{\tilde{\alpha}},
$$

with $J\left(\tilde{X}_{\bar{\alpha}}(g)\right)(\cdot) \in \mathscr{H}\left(X_{\tilde{\alpha}}\right)$. Similarly, it can be proved that $\mathscr{H}\left(\tilde{X}_{\tilde{\alpha}}\right)=U_{\bar{\alpha}}$.

Now, again using the $\tilde{\alpha}$-duality condition, and the definition of operators $S_{\tilde{\alpha}}$ and $S_{\tilde{\alpha}}^{\prime}$, we obtain, for each $\varphi \in U_{\tilde{\alpha}}$,

$$
\begin{aligned}
\left\langle\tilde{X}_{\tilde{\alpha}}(g), X_{\tilde{\alpha}}(\varphi)\right\rangle_{H\left(X_{\tilde{\alpha})}\right.} & =\left\langle g^{*}, \varphi\right\rangle_{U_{\tilde{\alpha}}}=\left\langle g, \varphi^{*}\right\rangle_{\left[U_{\tilde{\alpha}}\right]^{*}}=\varphi(g)=\left[J^{\prime}\left(X_{\tilde{\alpha}}(\varphi)\right)\right](g) \\
& =\left[S_{\tilde{\alpha}}^{\prime}\left(X_{\tilde{\alpha}}(\varphi)\right)\right](g),
\end{aligned}
$$

for all $g \in\left[U_{\tilde{\alpha}}\right]^{*}$. Thus, $S_{\tilde{\alpha}}^{\prime}\left(X_{\tilde{\alpha}}(\varphi)\right)=\varphi$, for each $\varphi \in U_{\tilde{\alpha}}$. Hence, $S_{\tilde{\alpha}}^{\prime} X_{\tilde{\alpha}}$ is the identity operator on $U_{\tilde{\alpha}}$.

Conversely, for each $Y \in H\left(\tilde{X}_{\tilde{\alpha}}\right)=H\left(X_{\tilde{\alpha}}\right), Y$ defines an element $\varphi_{Y}$ of $\mathscr{H}\left(\tilde{X}_{\tilde{\alpha}}\right)=$ $U_{\tilde{\alpha}}$ by $\varphi_{Y}(g)=\left(S_{\tilde{\alpha}}^{\prime} Y\right)(g)=J^{\prime} Y(g)=E Y \tilde{X}_{\tilde{\alpha}}(g)$, for all $g \in\left[U_{\tilde{\alpha}}\right]^{*}$. Then, we have

$$
\begin{aligned}
\left\langle\tilde{X}_{\bar{\alpha}}(g), X_{\tilde{\alpha}}\left(S_{\tilde{\alpha}}^{\prime} Y\right)\right\rangle_{H\left(X_{\bar{\alpha}}\right)} & =\left\langle\tilde{X}_{\bar{\alpha}}(g), X_{\tilde{\alpha}}\left(\varphi_{Y}\right)\right\rangle_{H\left(X_{\tilde{\alpha})}\right)}=\left\langle g, \varphi_{Y}^{*}\right\rangle_{\left[U_{\tilde{\alpha}}\right)^{*}} \\
& =E\left[\tilde{X}_{\tilde{\alpha}}(g) Y\right]=\left\langle\tilde{X}_{\tilde{\alpha}}(g), Y\right\rangle_{H\left(X_{\tilde{\alpha}}\right)^{*}}
\end{aligned}
$$

Hence, $\left(X_{\tilde{\alpha}}\left(S_{\tilde{\alpha}}^{\prime} Y\right)-Y\right)$ is orthogonal in $H\left(X_{\tilde{\alpha}}\right)$ to the range $\tilde{X}_{\tilde{\alpha}}\left(\left[U_{\tilde{\alpha}}\right]^{*}\right)$ of $\tilde{X}_{\tilde{\alpha}}$. As $\tilde{X}_{\tilde{\alpha}}\left(\left[U_{\tilde{\alpha}}\right]^{*}\right)$ is dense in $H\left(\tilde{X}_{\tilde{\alpha}}\right)=H\left(X_{\tilde{\alpha}}\right)$, we obtain that $X_{\tilde{\alpha}} S_{\tilde{\alpha}}^{\prime}$ is the identity operator on $H\left(X_{\tilde{\alpha}}\right)$.

Similarly, it is deduced that $S_{\tilde{\alpha}} \tilde{X}_{\tilde{\alpha}}$ and $\tilde{X}_{\tilde{\alpha}} S_{\tilde{\alpha}}$ are the identity operators on the spaces $\left[U_{\tilde{\alpha}}\right]^{*}$ and $H\left(X_{\tilde{\alpha}}\right)=H\left(\tilde{X}_{\tilde{\alpha}}\right)$, respectively.

(ii) By definition, the operators $J$ from $H\left(X_{\tilde{\alpha}}\right)$ onto $\mathscr{H}\left(X_{\bar{\alpha}}\right)$, and $J^{\prime}$ from $H\left(\tilde{X}_{\tilde{\alpha}}\right)$ onto $\mathscr{H}\left(\tilde{X}_{\tilde{\alpha}}\right)$ are bicontinuous. Therefore, we must only prove that the operators $\mathscr{K}$ from $\mathscr{H}\left(X_{\tilde{\alpha}}\right)$ onto $\left[U_{\tilde{\alpha}}\right]^{*}$, and $\mathscr{K}^{\prime}$ from $\mathscr{H}\left(\tilde{X}_{\tilde{\alpha}}\right)$ onto $U_{\tilde{\alpha}}$ are bicontinuous.

Let $\left\{\varphi_{n}\right\}_{n \in \mathbb{N}} \subseteq U_{\tilde{\alpha}}$ be a sequence convergent in the topology of $U_{\tilde{\alpha}}: \varphi_{n} \rightarrow\|\cdot\|_{U_{\tilde{a}}} \varphi \in U_{\tilde{\alpha}}$. Then, from the mean-square continuity of $X_{\bar{\alpha}}$ and from (i),

$$
\begin{aligned}
X_{\bar{\alpha}}\left(\varphi_{n}\right) \rightarrow\|\cdot\|_{H\left(X_{\tilde{\alpha}}\right)} X_{\tilde{\alpha}}(\varphi) & \Leftrightarrow J^{\prime} X_{\tilde{\alpha}}\left(\varphi_{n}\right) \rightarrow\|\cdot\|_{\mathscr{H}\left(\tilde{X}_{\tilde{\alpha})}\right)} J^{\prime} X_{\tilde{\alpha}}(\varphi) \\
& \Leftrightarrow\left[\mathscr{K}^{\prime}\right]^{-1}\left(\varphi_{n}\right) \rightarrow\|\cdot\|_{\mathscr{H}\left(\tilde{X}_{\tilde{\alpha})}\right)}\left[\mathscr{K}^{\prime}\right]^{-1}(\varphi) .
\end{aligned}
$$


Conversely, if $\left\{\varphi_{n}\right\}_{n \in \mathrm{N}}$ is a sequence convergent in $\mathscr{H}\left(\tilde{X}_{\tilde{\alpha}}\right), \varphi_{n} \rightarrow\|\cdot\|_{\mathscr{H}\left(\tilde{X}_{\tilde{\alpha}}\right)} \varphi \in \mathscr{H}\left(\tilde{X}_{\tilde{\alpha}}\right)$, we obtain, from (2.18),

$$
X_{\bar{\alpha}}\left(\mathscr{K}^{\prime} \varphi_{n}\right) \rightarrow\|\cdot\|_{H\left(X_{\dot{\alpha}}\right)} X_{\bar{\alpha}}\left(\mathscr{K}^{\prime} \varphi\right) \text {, that is, } X_{\bar{\alpha}}\left(\mathscr{K}^{\prime} \varphi_{n}-\mathscr{K}^{\prime} \varphi\right) \rightarrow\|\cdot\|_{H\left(X_{\dot{\alpha}}\right)} 0 \text {. }
$$

Equation (2.19) implies, under the duality condition,

$$
\begin{aligned}
0 & =\left\langle\lim _{n \rightarrow \infty} X_{\tilde{\alpha}}\left(\mathscr{K}^{\prime} \varphi_{n}-\mathscr{K}^{\prime} \varphi\right), \tilde{X}_{\tilde{\alpha}}(g)\right\rangle_{H\left(X_{\tilde{\alpha}}\right)} \\
& =\lim _{n \rightarrow \infty}\left\langle X_{\tilde{\alpha}}\left(\mathscr{K}^{\prime} \varphi_{n}-\mathscr{K}^{\prime} \varphi\right), \tilde{X}_{\bar{\alpha}}(g)\right\rangle_{H\left(X_{\tilde{\alpha})}\right)}=\lim _{n \rightarrow \infty}\left\langle\mathscr{K}^{\prime} \varphi_{n}-\mathscr{K}^{\prime} \varphi, g^{*}\right\rangle_{U_{\tilde{\alpha}}} \\
& =\left\langle\lim _{n \rightarrow \infty}\left(\mathscr{K}^{\prime} \varphi_{n}-\mathscr{K}^{\prime} \varphi\right), g^{*}\right\rangle_{U_{\tilde{\alpha}}}, \quad \forall g \in\left[U_{\tilde{\alpha}}\right]^{*} .
\end{aligned}
$$

Thus, $\mathscr{K}^{\prime} \varphi_{n} \rightarrow\|\cdot\|_{U_{\tilde{a}}} \mathscr{K}^{\prime} \varphi$. The bicontinuity of $\mathscr{K}$ can be similarly proved.

The covariance factorisation of $X_{\tilde{\alpha}}$ can be obtained in terms of the operators $S_{\tilde{\alpha}}$ and $S_{\tilde{\alpha}}^{\prime}$.

THEOREM 2.2. Let $X_{\alpha}$ be an $\alpha$-GRF with minimum fractional duality order $\tilde{\alpha}$. Then, the covariance operator $R_{\tilde{\alpha}}$ of $X_{\tilde{\alpha}}$ and the covariance operator $\tilde{R}_{\tilde{\alpha}}$ of $\tilde{X}_{\bar{\alpha}}$ can be factorised, respectively, as

$$
R_{\tilde{\alpha}}=S_{\tilde{\alpha}}\left(S_{\tilde{\alpha}}^{\prime}\right)^{-1}, \quad \tilde{R}_{\tilde{\alpha}}=S_{\tilde{\alpha}}^{\prime} S_{\tilde{\alpha}}^{-1} .
$$

PROOF. See Theorem 1 of Ruiz-Medina et al. [23].

The above covariance factorisation is the basis for an abstract representation of $X_{\tilde{\alpha}}$ in terms of generalised white noise.

DEFINITION 2.6. A generalised random field $\varepsilon(\cdot)$ defined on Hilbert space $\left(H,\langle\cdot, \cdot\rangle_{H}\right)$ is called a generalised white noise (GWN) relative to $H$ if

$$
\langle\varepsilon(u), \varepsilon(v)\rangle_{H(\varepsilon)}=\langle u, v\rangle_{H}, \quad \forall u, v \in H .
$$

DEFINITION 2.7. A generalised random field $X$ defined on a Hilbert space $\left(H,\langle\cdot, \cdot\rangle_{H}\right)$ is said to have a (weak-sense) abstract representation if there exists an isomorphism $L: H \rightarrow H$ such that

$$
\langle X L(u), X L(v)\rangle_{H(X)}=\langle u, v\rangle_{H}, \quad \forall u, v \in H,
$$

that is, if $\varepsilon \equiv X L$ is a GWN relative to $H$. This abstract representation is written as

$$
X(L u)=\varepsilon(u), \quad \forall u \in H .
$$


THEOREM 2.3. Assuming the existence of the $\tilde{\alpha}$-dual GRF $\tilde{X}_{\tilde{\alpha}}$ of the $\alpha$-GRF $X_{\alpha}$, with $\tilde{\alpha}$ being the minimum fractional duality order of $X_{\alpha}$, the restriction $X_{\tilde{\alpha}}$ to $U_{\tilde{\alpha}}$ of $X_{\alpha}$ has a weak-sense abstract representation, which is unique except for isometric isomorphisms.

PROOF. Let $\left\{Y_{n}\right\}_{n \in \mathbb{N}}$ and $\left\{\varphi_{n}\right\}_{n \in \mathbb{N}}$ be two orthonormal bases of the spaces $H\left(X_{\tilde{\alpha}}\right)=$ $H\left(\tilde{X}_{\tilde{\alpha}}\right)$ and $U_{\tilde{\alpha}}$, respectively, noting that $H\left(X_{\tilde{\alpha}}\right)$ and $U_{\tilde{\alpha}}$ are separable Hilbert spaces. We define the isometric isomorphism

$$
\mathscr{I}:\left(U_{\tilde{\alpha}},(\cdot, \cdot\rangle_{U_{\tilde{\alpha}}}\right) \rightarrow\left(H\left(X_{\tilde{\alpha}}\right),(\cdot, \cdot\rangle_{H\left(X_{\tilde{\alpha}}\right)}\right)
$$

by

$$
\varphi_{k} \rightarrow \mathscr{I} \varphi_{k}=Y_{k}, \quad \forall k \in \mathbb{N} .
$$

Then, considering the isomorphism $L$ on $U_{\tilde{\alpha}}$ defined as $L:=S_{\tilde{\alpha}}^{\prime} \mathscr{I}$, with $S_{\tilde{\alpha}}^{\prime}$ being the operator defined above, we obtain, from Proposition 2.1,

$$
\begin{aligned}
\left\langle X_{\tilde{\alpha}} L(\phi), X_{\tilde{\alpha}} L(\varphi)\right\rangle_{H\left(X_{\dot{\alpha})}\right)} & =\left\langle X_{\tilde{\alpha}} S_{\tilde{\alpha}}^{\prime} \mathscr{I}(\phi), X_{\tilde{\alpha}} S_{\tilde{\alpha}}^{\prime} \mathscr{I}(\varphi)\right\rangle_{H\left(X_{\tilde{\alpha})}\right)} \\
& =\langle\mathscr{I}(\phi), \mathscr{I}(\varphi)\rangle_{H\left(X_{\dot{\alpha}}\right)}=\langle\phi, \psi\rangle_{U_{\tilde{\alpha}}}, \quad \forall \phi, \varphi \in U_{\tilde{\alpha}} .
\end{aligned}
$$

Let us now assume that

$$
\begin{aligned}
X_{\tilde{\alpha}}\left(L_{1} \phi\right) & =\varepsilon_{\tilde{\alpha}, 1}(\phi), \quad \forall \phi \in U_{\tilde{\alpha}}, \\
X_{\tilde{\alpha}}\left(L_{2} \psi\right) & =\varepsilon_{\tilde{\alpha}, 2}(\psi), \quad \forall \psi \in U_{\tilde{\alpha}},
\end{aligned}
$$

with $L_{1}, L_{2}$ being isomorphisms on $U_{\tilde{\alpha}}$ and $\varepsilon_{\tilde{\alpha}, 1}, \varepsilon_{\tilde{\alpha}, 2}$ being GWNs relative to $U_{\tilde{\alpha}}$. Then, with $V=L_{1}^{-1} L_{2}$,

$$
\begin{aligned}
\langle V \phi, V \psi\rangle_{U_{\tilde{\alpha}}} & =\left\langle L_{1}^{-1} L_{2} \phi, L_{1}^{-1} L_{2} \psi\right\rangle_{U_{\tilde{\alpha}}}=\left\langle X_{\tilde{\alpha}}\left(L_{1} L_{1}^{-1} L_{2} \phi\right), X_{\tilde{\alpha}}\left(L_{1} L_{1}^{-1} L_{2} \psi\right)\right\rangle_{\mathscr{L}^{2}(\Omega)} \\
& =\left\langle X_{\tilde{\alpha}}\left(L_{2} \phi\right), X_{\tilde{\alpha}}\left(L_{2} \psi\right)\right\rangle_{\mathscr{L}^{2}(\Omega)}=\langle\phi, \psi\rangle_{U_{\tilde{\alpha}}}, \quad \forall \phi, \psi \in U_{\tilde{\alpha}} .
\end{aligned}
$$

The above abstract representation is written as

$$
X_{\tilde{\alpha}}(L \phi)=\varepsilon_{\tilde{\alpha}}(\phi), \quad \forall \phi \in U_{\tilde{\alpha}} .
$$

Then

$$
\left\langle X_{\tilde{\alpha}}(L \phi), X_{\tilde{\alpha}}(L \psi)\right\rangle_{H\left(X_{\tilde{\alpha}}\right)}=\left\langle\varepsilon_{\tilde{\alpha}}(\phi), \varepsilon_{\tilde{\alpha}}(\psi)\right\rangle_{H\left(X_{\tilde{\alpha}}\right)}=\langle\phi, \psi\rangle_{U_{\tilde{a}}}, \quad \forall \phi, \psi \in U_{\tilde{\alpha}} .
$$

Since

$$
\langle\phi, \psi\rangle_{U_{\tilde{\sigma}}}=\left\langle\mathscr{I}_{\tilde{\alpha}}(\phi), \mathscr{I}_{\tilde{\alpha}}(\varphi)\right\rangle_{L^{2}(T)}, \quad \forall \phi, \psi \in U_{\tilde{\alpha}},
$$

where $\mathscr{I}_{\tilde{\alpha}}$ is the inverse of the Bessel potential $\mathscr{I}_{-\tilde{\alpha}}$ (defined as $\mathscr{I}_{-\tilde{\alpha}}=(I-\Delta)^{-\tilde{\alpha} / 2}, I=$ identity operator, $\Delta=$ Laplacian operator), we also obtain the following interpretation of the representation (2.20):

$$
X_{\tilde{\alpha}}(L \phi)=\varepsilon_{L^{2}(T)} \mathscr{I}_{\tilde{\alpha}}(\phi), \quad \forall \phi \in U_{\tilde{\alpha}},
$$


where $\varepsilon_{L^{2}(T)}(\cdot)$ is a GWN relative to $L^{2}(T)$. The right hand side of (2.21) represents the weak-sense derivative of fractional order $\tilde{\alpha}$ of a GWN in $L^{2}(T)$.

In a similar fashion, the condition of Theorem 2.2 also implies a unique abstract representation for the $\tilde{\alpha}$-dual GRF $\tilde{X}_{\bar{\alpha}}$ :

$$
\tilde{X}_{\tilde{\alpha}}(\tilde{L} g)=\tilde{\varepsilon}_{\tilde{\alpha}}(g), \quad \forall g \in\left[U_{\tilde{\alpha}}\right]^{*},
$$

with $\tilde{L}=R_{\bar{\alpha}} L I_{\left[U_{\tilde{\alpha}}\right]}^{*}, R_{\bar{\alpha}}$ being the covariance operator of $X_{\bar{\alpha}}, L$ being the isomorphism defining the abstract representation of $X_{\tilde{\alpha}}$, and $I_{\left[U_{\tilde{\alpha}}\right]^{*}}:\left[U_{\tilde{\alpha}}\right]^{*} \rightarrow U_{\tilde{\alpha}}$ being the isometric isomorphism defined by the Riesz representation theorem.

Similar to (2.21), we can also write (2.22) alternatively as

$$
\tilde{X}_{\tilde{\alpha}}(\tilde{L} g)=\tilde{\varepsilon}_{L^{2}(T)} \mathscr{I}_{-\tilde{\alpha}}(g), \quad \forall g \in\left[U_{\tilde{\alpha}}\right]^{*} .
$$

The right hand side of (2.23) is interpreted as weak-sense fractional integration of a GWN relative to $L^{2}(T)$.

The abstract representation (2.21) can be equivalently expressed as

$$
X_{\tilde{\alpha}}(\phi)=\varepsilon_{L^{2}(T)}\left[\mathscr{I}_{\tilde{\alpha}}\left(L^{-1} \phi\right)\right], \quad \forall \phi \in U_{\tilde{\alpha}} .
$$

The generalised covariance function $B_{\tilde{\alpha}}$ of the $\tilde{\alpha}$-GRF $X_{\tilde{\alpha}}$ then takes the form

$$
\begin{aligned}
B_{\tilde{\alpha}}(\phi, \varphi) & =E\left[X_{\tilde{\alpha}}(\phi) X_{\tilde{\alpha}}(\varphi)\right]=\left\langle\mathscr{I}_{\tilde{\alpha}} L^{-1}(\phi), \mathscr{I}_{\tilde{\alpha}} L^{-1}(\varphi)\right\rangle_{L^{2}(T)} \\
& =\left\langle\phi^{*},\left[L^{-1}\right]^{*} \mathscr{I}_{\tilde{\alpha}}^{*} \mathscr{I}_{\tilde{\alpha}} L^{-1}(\varphi)\right\rangle_{\left[U_{\tilde{\alpha}}\right]^{*}}=\left\langle\phi^{*}, R_{\tilde{\alpha}}(\varphi)\right\rangle_{\left[U_{\tilde{\alpha}}\right]^{*}}, \quad \forall \phi, \varphi \in U_{\tilde{\alpha}} .
\end{aligned}
$$

Consequently, the covariance operator $R_{\tilde{\alpha}}$ of $X_{\tilde{\alpha}}$ is given by

$$
R_{\bar{\alpha}}=\left[\mathscr{I}_{\tilde{\alpha}} L^{-1}\right]^{*}\left[\mathscr{I}_{\tilde{\alpha}} L^{-1}\right] \text {. }
$$

In a similar fashion, the covariance operator $\tilde{R}_{\tilde{\alpha}}$ of $\tilde{X}_{\tilde{\alpha}}$ is given by

\begin{tabular}{|c|c|c|c|}
\hline $\begin{array}{c}\text { Hilbert } \\
\text { space }\end{array}$ & $\begin{array}{c}\text { Inner } \\
\text { product }\end{array}$ & $\begin{array}{l}\text { Dual } \\
\text { space }\end{array}$ & $\begin{array}{l}\text { Isomorphic mapping }(\cong) \\
\text { Isometric mapping }(\equiv)\end{array}$ \\
\hline $\bar{H}^{\alpha}(T)$ & $\langle\phi, \varphi\rangle_{\bar{H}^{\alpha}(T)}=\left\langle\mathscr{I}_{\alpha}(\phi), \mathscr{I}_{\alpha}(\varphi)\right\rangle_{L^{2}(T)}$ & $H^{-\alpha}(T)$ & $\bar{H}^{\alpha}(T) \simeq \mathscr{H}\left(\tilde{X}_{\alpha}\right)$ \\
\hline $\mathscr{H}\left(X_{\alpha}\right)$ & $\langle u, v\rangle_{\mathscr{H}\left(X_{\alpha}\right)}=E\left[X_{u} X_{v}\right]$ & $\mathscr{H}\left(\tilde{X}_{\alpha}\right)$ & $\mathscr{H}\left(X_{\alpha}\right) \simeq H^{-\alpha}(T)$ \\
\hline$H\left(X_{\alpha}\right)$ & $\langle X, Y\rangle_{H\left(X_{\alpha}\right)}=E[X Y]$ & $H\left(\tilde{X}_{\alpha}\right)$ & $H\left(X_{\alpha}\right) \equiv \mathscr{H}\left(X_{\alpha}\right)$ \\
\hline
\end{tabular}

$$
\tilde{R}_{\bar{\alpha}}=\left[\mathscr{I}_{-\bar{\alpha}} \tilde{L}^{-1}\right]^{*}\left[\mathscr{I}_{-\bar{\alpha}} \tilde{L}^{-1}\right] \text {. }
$$

In view of the above definitions and Proposition 2.1, the following table summarises the fundamental Hilbert spaces involved in the development of the fractional generalised framework. 
REMARK 2.5. Note that in the above table we have assumed that the GRF $X_{\alpha}$ has minimum fractional duality order $\alpha$. In the case where the minimum fractional duality order $\tilde{\alpha}$ is larger than $\alpha$, this table can be directly rewritten in terms of the restriction $X_{\tilde{\alpha}}$ of $X_{\alpha}$ to the space $\bar{H}^{\tilde{\alpha}}(T)$.

We now outline some fundamental results about the spectral representation of selfadjoint operators defined on a separable Hilbert space $H$ (see, for example, Dautray and Lions [11]). These results are needed in Section 4.

For a compact and self-adjoint operator $A$ defined on a separable Hilbert space $H$, the Hilbert-Schmidt Theorem provides a decomposition of the space $H$ into a direct sum of the eigenspaces $V_{k}, k \in \mathbb{N}$, corresponding to its eigenvalues $\lambda_{k} \in \mathbb{R}, k \in \mathbb{N}$ (including the eigenspace $V_{0}$ associated with the eigenvalue 0 ):

Furthermore,

$$
H=\bigoplus_{k \in N} V_{k}
$$

$$
A u=\sum_{k \in \mathbb{N}} \lambda_{k} P_{k}(u)=\sum_{k \in \mathbb{N}} \lambda_{k}\left\langle u, u_{k}\right\rangle_{H} u_{k}, \quad \forall u \in H,
$$

with $A u_{k}=\lambda_{k} u_{k}, u_{k} \in V_{k}, \forall k \in \mathbb{N}$, and

$$
I=\sum_{k \in \mathrm{N}} P_{k},
$$

where we denote by $P_{k}$ the projection operator into the eigenspace $V_{k}$, by $\langle\cdot, \cdot\rangle_{H}$ the inner product in the space $H$, and by $I$ the identity operator.

For each $\lambda \in \mathbb{R}$, the following spaces and operators are defined:

$$
\begin{aligned}
G_{\lambda} & =\bigoplus_{\lambda_{k} \leq \lambda} V_{k}, \\
E_{\lambda} & \equiv \text { orthogonal projection onto } G_{\lambda} .
\end{aligned}
$$

A family $\left\{E_{\lambda}\right\}_{\lambda \in R}$ of orthogonal projections in a separable Hilbert space $H$ is called a spectral family (or a resolution of the identity) if it satisfies the following conditions:

(i) $E_{\lambda} \cdot E_{\mu}=E_{\text {inf }\{\lambda, \mu\}}$.

(ii) For all $\lambda \in \mathbb{R}, E_{\lambda+0}=E_{\lambda}$.

(iii) $\lim _{\lambda \rightarrow-\infty} E_{\lambda}=0, \lim _{\lambda \rightarrow \infty} E_{\lambda}=I$.

Clearly, $\left\{E_{\lambda}\right\}_{\lambda \in \mathbb{R}}$ defined in (2.28) is a spectral family. In this case, as $A$ is a compact and self-adjoint operator, $\sigma(A)$ is a bounded set in $\mathbb{R}$, and there exist constants $a, b \in \mathbb{R}$ such that $E_{\lambda}=0$ for $\lambda<a$, and $E_{\lambda}=I$ for $\lambda \geq b$. Furthermore, the discontinuities of the function $\lambda \rightarrow E_{\lambda}$ are the eigenvalues $\lambda_{k}, E_{\lambda_{k}}-E_{\lambda_{k}-0}=P_{k}, \forall k \in \mathbb{N}$, and in the sense of distributions in $\mathbb{R}$ with values in $\mathscr{L}(H)$, the derivative of $E_{\lambda}$ can be identified 
with a measure $d E_{\lambda}$ defined by

$$
d E_{\lambda}=\sum_{\lambda_{k} \leq \lambda} \delta_{\lambda_{k}} \otimes P_{k}
$$

(see Dautray and Lions [11, page 112]).

The following result establishes the spectral representatiom of a self-adjoint operator $A$ on $H$ as well as the spectral representation of certain functions of such an operator:

THEOREM 2.4. Let $H$ be a separable complex Hilbert space.

(i) There exists a bijective mapping $\hat{\sigma}$ from the set of spectral families in $H$ onto the set of self-adjoint operators on $H$.

(ii) Let $A$ be the self-adjoint operator associated with a spectral family $\left\{E_{\lambda}\right\}_{\lambda \in R}$ (under the mapping $\hat{\sigma}$ ). Then, we have that the domain of $A^{n}$ is

$$
D\left(A^{n}\right)=\left\{x \in H: \int_{-\infty}^{\infty} \lambda^{2 n} d\left(E_{\lambda} x, x\right)<\infty\right\}, \quad n \geq 1,
$$

and for $x \in D\left(A^{n}\right), y \in H$,

$$
\begin{aligned}
\left\langle A^{n} x, y\right\rangle_{H} & =\int_{-\infty}^{\infty} \lambda^{n} d\left(E_{\lambda} x, y\right), \\
\left\|A^{n} x\right\|_{H}^{2} & =\int_{-\infty}^{\infty} \lambda^{2 n} d\left(E_{\lambda} x, x\right) .
\end{aligned}
$$

(iii) If $P_{n}(\lambda)$ is a polynomial of degree $n$, then $P_{n}(A)$ is defined as

$$
\left\langle P_{n}(A) x, y\right\rangle_{H}=\int_{-\infty}^{\infty} P_{n}(\lambda) d\left(E_{\lambda} x, y\right), \quad \forall x \in D\left(A^{n}\right), \forall y \in H .
$$

(iv) If $f$ is a continuous function on $\mathbb{R}$, then

$$
\langle f(A) x, y\rangle_{H}=\int_{-\infty}^{\infty} f(\lambda) d\left(E_{\lambda} x, y\right), \quad \forall x \in D(f(A)), \forall y \in H .
$$

The integrals appearing in (2.29)-(2.33) are understood as improper operator Stieltjes integrals which converge strongly.

PROOF. See Dautray and Lions [11, pages 119 and 126].

In addition, the next theorem provides a functional calculus for functions of selfadjoint operators which are not necessarily bounded. 
THEOREM 2.5. Let A be a self-adjoint operator in the separable complex Hilbert space $H$.

(i) If $\bar{f}$ is the complex conjugate function for $f$, then

$$
D(\bar{f}(A))=D(f(A)),
$$

and for all $x, y \in D(f(A))$,

$$
\langle f(A) x, y\rangle_{H}=\left\langle x^{*}, \tilde{f}(A) y^{*}\right\rangle .
$$

(ii) If $x \in D(f(A)), y \in D(g(A))$, then

$$
\langle f(A) x, g(A) y\rangle_{H}=\int_{-\infty}^{\infty} f(\lambda) \bar{g}(\lambda) d\left(E_{\lambda} x, y\right) .
$$

(iii) For $\alpha \in \mathbb{C}, x \in D(f(A))$,

$$
(\alpha f)(A) x=\alpha f(A) x .
$$

For $x \in D(f(A)) \cap D(g(A))$, we have

$$
(f+g)(A) x=f(A) x+g(A) x .
$$

(iv) If $x \in D(f(A))$, then the condition $f(A) x \in D(g(A))$ is equivalent to the condition $x \in D[(g \circ f)(A)]$ (where $(g \circ f)(\lambda)=g(\lambda) f(\lambda)$,) and we have

$$
[g(A) f(A)] x=(g \circ f)(A) x .
$$

(v) If $f \in \mathscr{M}_{0}$, with $\mathscr{M}_{0}$ being the class of functions which are measurable with respect to $d_{\lambda}\left(E_{\lambda} x, x\right)$, for all $x \in H$, and $D(f(A))$ is dense in $H$, then the adjoint $[f(A)]^{\prime}$ of $f(A)$ satisfies

$$
[f(A)]^{\prime}=\bar{f}(A)
$$

$f(A)$ is then normal (and self-adjoint whenever $\bar{f}=f$ ).

(vi) If $\neq 0$ almost everywhere with respect to the measure $\left\{\sigma_{x}\right\}_{x \in H}$, then $[f(A)]^{-1}$ exists and

$$
[f(A)]^{-1}=(1 / f)(A) .
$$

ProOF. See Dautray and Lions [11, page 140].

The projection operator $E_{\lambda}$ of the spectral family of a self-adjoint operator $A$ on a separable Hilbert space $H$ can be represented as an integral operator with kernel $E(\cdot, \cdot, \lambda)$ defined as follows (see, for example, Ramm [21, pages 145-148]):

$$
E(\mathbf{z}, \mathbf{y}, \lambda)=\int_{-\infty}^{\lambda} \Phi(\mathbf{z}, \mathbf{y}, \lambda) d \rho(\lambda)
$$


where $\Phi$ is the generalised spectral kernel and $\rho(\cdot)$ is the spectral measure associated with $A$. Then, for a Borel measurable function $F, F(A)$ can be represented as an integral operator with kernel

$$
F(A)(\mathbf{y}, \mathbf{z})=\int_{-\infty}^{\infty} F(\lambda) \Phi(\mathbf{y}, \mathbf{z}, \lambda) d \rho(\lambda)
$$

\section{Estimation of fractional generalised random fields}

We consider the ordinary least-squares linear estimation problem introduced in Section 1. The duality condition relative to a fractional Sobolev space (see Definition 2.5) leads to a fractional weak-sense solution to this problem. More specifically, we study the minimum fractional duality order of the fractional generalised ordinary random field defined by the information random field $\mathscr{X}$. This order allows us to determine the fractional Sobolev space where the inverse of the covariance operator of $\mathscr{X}$ can be defined as a bounded operator. Hence, the solution with minimum fractional singularity order belongs to the dual of such a fractional Sobolev space, and its singularity order coincides with the minimum fractional duality order of the information random field.

As commented in the Introduction, the approach of Ramm [21] is applied to a class of ordinary random fields with covariance operators in the class $\mathscr{R}$ defined by (1.5). In particular, this class satisfies the duality condition introduced in Definition 2.5, since each element of $\mathscr{R}$ defines an isomorphism between the fractional Sobolev spaces $\bar{H}^{-(q-p) s / 2}(T)$ and $H^{(q-p) s / 2}(T)$ (see Ramm [21, Theorem 1, page 12]). That is, the minimum fractional duality order of the corresponding generalised ordinary random field is $\alpha=(q-p) s / 2$ (see Remark 2.4), which coincides with the singularity order of the solution calculated by using this approach.

Furthermore, although the fractional generalised approach we present here is concerned with the regularisation of least-squares linear estimation problems of systems involving second-order ordinary random fields (see Remark 2.3), this approach also provides a solution for the case where the system involves random fields with positive minimum fractional singularity order, that is, improper random fields.

Let $\mathscr{S}$ and $\mathscr{Y}$ be two zero-mean second-order random fields related by the following equation in the mean-square sense:

$$
(A \mathscr{S})(\mathbf{z})=\mathscr{Y}(\mathbf{z}), \quad \forall \mathbf{z} \in T \subseteq \mathbb{R}^{d},
$$

where $A$ is a known operator which can be linear or non-linear. Assuming that $\mathscr{S}$ and $\mathscr{Y}$ have minimum fractional singularity orders $\gamma \in \mathbb{Q}$ and $\beta \in \mathbb{Q}$, respectively, we can consider the associated fractional generalised ordinary random fields $\mathscr{S}_{\gamma}$ on 
$\bar{H}^{\gamma}(T)=U_{\gamma}$ and $\mathscr{Y}_{\beta}$ on $\bar{H}^{\beta}(T)=U_{\beta}$. The fractional generalised version of (3.1) is then given by

$$
\mathscr{Y}_{\beta}(\phi)=\mathscr{S}_{\gamma}\left(A^{\prime} \phi\right), \quad \forall \phi \in U_{\beta},
$$

where $A^{\prime}$ represents the adjoint operator of $A$, defined from $U_{\beta}$ into $U_{\gamma}$. The estimation problem in this fractional generalised framework then consists of calculating the leastsquares linear estimate of the fractional generalised output $\mathscr{Y}_{\beta}$ from the information provided by the fractional generalised version of (1.2) given by

$$
\mathscr{X}_{\alpha}(\varphi)=S_{\gamma}(\varphi)+\mathscr{N}_{\theta}(\varphi), \quad \forall \varphi \in U_{\alpha},
$$

where $\mathscr{N}_{\theta}$ denotes the fractional generalised ordinary random field defined by the observation noise $\mathscr{N}$ with minimum fractional singularity order $\theta \in \mathbb{Q}$, and $\alpha \in$ $\mathbb{Q}$ represents the minimum fractional singularity order of the ordinary information random field $\mathscr{X}$ defining the fractional generalised ordinary random field $\mathscr{X}_{\alpha}$ on $\bar{H}^{\alpha}(T)=U_{\alpha}$. Note that $\alpha$ can be taken to be the maximum between $\gamma$ and $\theta$. In fact, if $\mathscr{X}_{\alpha}$ has minimum fractional duality order $\tilde{\alpha} \geq \alpha$, then the information used in the calculation of the solution to the estimation problem in a fractional generalised framework is provided by the observation of $\mathscr{X}_{\alpha}$ on $U_{\tilde{\alpha}}$, that is, the useful information is represented by the restriction to $U_{\tilde{\alpha}}$ of $\mathscr{X}_{\alpha}$ given by

$$
\mathscr{X}_{\tilde{\alpha}}(\varphi)=\mathscr{S}_{\gamma}(\varphi)+\mathscr{N}_{\theta}(\varphi), \quad \forall \varphi \in U_{\tilde{\alpha}}
$$

We also assume that the covariance function $B_{\mathscr{X}_{\tilde{\alpha}}}$ of $\mathscr{X}_{\tilde{\alpha}}$ and the cross-covariance function $B_{\mathscr{X}_{\tilde{\alpha}} \mathscr{Y}_{\beta}}$ between $\mathscr{X}_{\tilde{\alpha}}$ and $\mathscr{Y}_{\beta}$ are known. The least-squares linear estimate

$$
\hat{\mathscr{Y}}_{\beta}(\phi)=\mathscr{X}_{\tilde{\alpha}}\left(K^{\prime} \phi\right), \quad \forall \phi \in U_{\beta},
$$

of $\mathscr{Y}_{\beta}$ is then obtained by minimising the least-squares error

$$
\varepsilon\left(K^{\prime} \phi\right):=E\left[\mathscr{Y}_{\beta}(\phi)-\mathscr{X}_{\dot{\alpha}}\left(K^{\prime}(\phi)\right)\right]^{2},
$$

for all $\phi \in U_{\beta}$, where $K^{\prime}$ is a linear operator defined from $U_{\beta}$ into $U_{\bar{\alpha}}$, and represents the adjoint of the integral operator $K$ with kernel $k(\cdot, \cdot)$ defined as in (1.3). Hence, the solution $k(\cdot, \cdot)$ to the least-squares linear estimation problem can be found in the space $V_{\beta} \otimes U_{\tilde{\alpha}}$. If $A$ is a differential operator, then $\beta \leq \gamma$, and if $A$ is an integral operator, then $\gamma \leq \beta$. In the case when $A$ is a shift operator, we have the extrapolation problem, and when $A$ is the identity operator, we have the filtering problem. 
The covariance functions $B_{\mathscr{X}_{\tilde{\alpha}}}$ of $\mathscr{X}_{\tilde{\alpha}}$ and $B_{\mathscr{S}_{Y}}$ of $\mathscr{S}_{Y}$, and the cross-covariance function $B_{\mathscr{X}_{\tilde{\alpha}} \mathscr{\mathscr { S }}_{\beta}}$ between $\mathscr{X}_{\tilde{\alpha}}$ and $\mathscr{S}_{\gamma}$ are given by the following expressions:

$$
\begin{aligned}
& B_{\mathscr{X}_{\tilde{\boldsymbol{\alpha}}}}(\varphi, \psi)=\left\langle\left[R_{\mathscr{X}_{\tilde{\boldsymbol{\alpha}}}}(\varphi)\right]^{*}, \psi\right\rangle_{U_{\tilde{\alpha}}}, \quad \forall \varphi, \psi \in U_{\tilde{\alpha}}, \\
& B_{\mathscr{S}_{r}}(\varphi, \psi)=\left\langle\left[R_{\mathscr{S}_{r}}(\varphi)\right]^{*}, \psi\right\rangle_{U_{\gamma}} \quad \forall \varphi, \psi \in U_{\gamma}, \\
& B_{\mathscr{X}_{\tilde{\alpha}} \mathscr{Y}_{\beta}}(\varphi, \phi)=E\left[\mathscr{X}_{\tilde{\alpha}}(\varphi) \mathscr{S}_{\gamma}\left(A^{\prime} \phi\right)\right] \\
& =B_{\mathscr{X}_{\dot{\alpha}} \mathscr{S}_{\gamma}}\left(\varphi, A^{\prime} \phi\right)=\left\langle\left[R_{\mathscr{X}_{\dot{\alpha}} \mathscr{S}_{\nu}}^{\prime}(\varphi)\right]^{*}, A^{\prime}(\phi)\right\rangle_{v_{y}} \\
& =\left\langle\varphi,\left[R_{\mathscr{X}_{\tilde{\alpha}} \mathscr{S}_{\gamma}}\left(A^{\prime} \phi\right)\right]^{*}\right\rangle_{U_{\hat{\alpha}}}, \quad \forall \varphi \in U_{\tilde{\alpha}}, \phi \in U_{\beta},
\end{aligned}
$$

where $R_{\mathscr{X}_{\tilde{\alpha}}}: U_{\tilde{\alpha}} \rightarrow\left[U_{\tilde{\alpha}}\right]^{*}=V_{\tilde{\alpha}}, R_{\mathscr{S}_{\gamma}}: U_{\gamma} \rightarrow\left[U_{\gamma}\right]^{*}$, and $R_{\mathscr{X}_{\tilde{\alpha}} \mathscr{S}_{\gamma}}: U_{\gamma} \rightarrow\left[U_{\tilde{\alpha}}\right]^{*}=V_{\tilde{\alpha}}$ denote the covariance operators associated, respectively, with the covariance functions

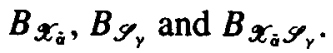

REMARK 3.1. Note that in the case where $R_{\mathscr{X}_{\tilde{\alpha}}} \in \mathscr{R}, k(\mathbf{z}, \cdot)$ belongs to $U_{\tilde{\alpha}}$, for each $\mathbf{z} \in T$, with $\tilde{\alpha}=-[(q-p) s / 2]$ for certain $s \in \mathbb{N}$ and $q>p>0$.

The least-squares error $\varepsilon$ associated with $K^{\prime}$, for each $\phi \in U_{\beta}$, is calculated from (3.5) and is given by

$$
\begin{aligned}
\varepsilon\left(K^{\prime} \phi\right)= & E\left[\mathscr{Y}_{\beta}(\phi)-\mathscr{X}_{\tilde{\alpha}}\left(K^{\prime}(\phi)\right)\right]^{2} \\
= & B_{\mathscr{X}_{\tilde{\alpha}}}\left(K^{\prime}(\phi), K^{\prime}(\phi)\right)+B_{\mathscr{S}_{r}}\left(A^{\prime} \phi, A^{\prime} \phi\right)-2 B_{\mathscr{X}_{\tilde{\alpha}} \mathscr{S}_{r}}\left(K^{\prime}(\phi), A^{\prime} \phi\right) \\
= & \left\langle\left[R_{\mathscr{X}_{\dot{\alpha}}}\left(K^{\prime}(\phi)\right)\right]^{*}, K^{\prime}(\phi)\right\rangle_{U_{\tilde{\alpha}}}+\left\langle\left[R_{\mathscr{S}_{\gamma}}\left(A^{\prime} \phi\right)\right]^{*}, A^{\prime}(\phi)\right\rangle_{U_{\gamma}} \\
& -2\left\langle K^{\prime}(\phi),\left[R_{\mathscr{X}_{\tilde{\alpha}} \mathscr{S}_{r}} A^{\prime}(\phi)\right]^{*}\right\rangle_{U_{\tilde{\alpha}}} .
\end{aligned}
$$

From the Orthogonal Projection Theorem, $K^{\prime}$ minimising $\varepsilon$ must satisfy, for $\phi \in U_{\beta}$,

$$
E\left[\left(\mathscr{Y}_{\beta}(\phi)-\mathscr{X}_{\bar{\alpha}}\left(K^{\prime} \phi\right) \mathscr{X}_{\bar{\alpha}}(\varphi)\right]=0, \quad \forall \varphi \in U_{\bar{\alpha}},\right.
$$

or equivalently,

$$
B_{\mathscr{Y}_{\hat{\beta}} \mathscr{X}_{\dot{\alpha}}}(\phi, \varphi)=B_{\mathscr{S}_{r} \mathscr{X}_{\dot{\alpha}}}\left(A^{\prime} \phi, \varphi\right)=B_{\mathscr{X}_{\tilde{\alpha}}}\left(K^{\prime} \phi, \varphi\right),
$$

for all $\varphi \in U_{\tilde{\alpha}}$. From (3.6), (3.8) can be rewritten as

$$
R_{\mathscr{X}_{\tilde{\alpha}} \mathscr{S}_{r}}\left(A^{\prime} \phi\right)(\varphi)=R_{\mathscr{X}_{\tilde{\alpha}}}\left(K^{\prime} \phi\right)(\varphi), \quad \forall \varphi \in U_{\tilde{\alpha}}
$$

That is,

$$
R_{\mathscr{X}_{\tilde{\alpha}} \mathscr{S}_{\bar{\gamma}}}\left(A^{\prime} \phi\right) \underset{\overline{U_{\tilde{\alpha}}}}{=} R_{\mathscr{X}_{\dot{\alpha}}}\left(K^{\prime} \phi\right),
$$

for each $\phi \in U_{\beta}$. Assuming that condition (3.10) holds, the partial derivative $\partial \varepsilon\left(K^{\prime} \phi+\xi \mathscr{L}\right) / \partial \xi$ is equal to zero for $\xi=0$, with $\xi$ a small real number, and 
$\mathscr{L}$ a linear operator defined from $U_{\beta}$ into $U_{\tilde{\alpha}}$. That is,

$$
\begin{aligned}
\varepsilon\left(\left(K^{\prime}+\xi \mathscr{L}\right)(\phi)\right)= & B_{\mathscr{X}_{\tilde{\dot{x}}}}\left(K^{\prime} \phi, K^{\prime} \phi\right)+2 \xi B_{\mathscr{X}_{\dot{\alpha}}}\left(\mathscr{L} \phi, K^{\prime} \phi\right) \\
& +\xi^{2} B_{\mathscr{X}_{\tilde{\alpha}}}(\mathscr{L} \phi, \mathscr{L} \phi)+B_{\mathscr{S}_{r}}\left(A^{\prime} \phi, A^{\prime} \phi\right) \\
& -2 B_{\mathscr{X}_{\tilde{\alpha}} \mathscr{Y}_{r}}\left(K^{\prime} \phi, A^{\prime} \phi\right)-2 \xi B_{\mathscr{X}_{\tilde{z}} \mathscr{S}_{r}}\left(\mathscr{L} \phi, A^{\prime} \phi\right)
\end{aligned}
$$

Then,

$$
\begin{aligned}
\frac{\partial \varepsilon\left(K^{\prime}+\xi \mathscr{L}\right)}{\partial \xi}= & 2\left\langle\left[R_{\mathscr{X}_{\dot{\alpha}}}\left(K^{\prime} \phi\right)\right]^{*}, \mathscr{L} \phi\right\rangle_{U_{\dot{\alpha}}}-2\left\langle\mathscr{L} \phi,\left[R_{\mathscr{X}_{\dot{\alpha}} \mathscr{S}_{\nu}}\left(A^{\prime} \phi\right)\right]^{*}\right\rangle_{U_{\tilde{\alpha}}} \\
& +2 \xi\left\langle\left[R_{\mathscr{X}_{\dot{\alpha}}}(\mathscr{L} \phi)\right]^{*}, \mathscr{L} \phi\right\rangle_{U_{\tilde{\alpha}}}, \quad \forall \phi \in U_{\beta},
\end{aligned}
$$

and (3.12) is null for $\xi=0$ if condition (3.10) holds. Similarly, from (3.8), the following adjoint equation is obtained for operator $K$ :

$$
K R_{\mathscr{X}_{\tilde{\alpha}}}(\varphi) \underset{U_{\tilde{\beta}}}{=} A R_{\mathscr{S}_{\gamma} \mathscr{x}_{\tilde{\alpha}}}(\varphi), \quad \forall \varphi \in U_{\tilde{\alpha}}
$$

This equation provides a fractional generalised version of (1.4) defining $k(\cdot, \cdot)$ in the ordinary case. Note that the adjoint equation of $(1.4)$ is also used to calculate $k(\cdot, \cdot)$ in the ordinary case (see, for example, Ramm [21]). Therefore, in the remainder of this section, we consider condition (3.10) or, equivalently the adjoint condition (3.13). In particular, assuming any of these conditions, the least squares error $\varepsilon$ is given by

$$
\varepsilon\left(K^{\prime} \phi\right)=\left\langle\left[R_{\mathscr{S}_{Y}}\left(A^{\prime} \phi\right)\right]^{*}, A^{\prime}(\phi)\right\rangle_{U_{y}}-\left\langle\left[R_{\mathscr{X}_{\tilde{a}}}\left(K^{\prime} \phi\right)\right]^{*}, K^{\prime}(\phi)\right\rangle_{U_{\tilde{\alpha}}} .
$$

Note that by definition of $K^{\prime}$, and since $\mathscr{H}\left(\tilde{\mathscr{X}}_{\tilde{\alpha}}\right)=\left(U_{\tilde{\alpha}},\langle\cdot, \cdot\rangle_{B_{\boldsymbol{x}_{\tilde{\alpha}}}}\right)$, we have

$$
B_{\mathscr{X}_{\hat{\alpha}}}\left(K^{\prime} \phi, K^{\prime} \phi\right)=\left\|K^{\prime} \phi\right\|_{\mathscr{C}_{(}\left(\tilde{\mathscr{X}}_{\dot{\underline{x}})}\right.}^{2}<\infty
$$

for all $\phi \in U_{\beta}$.

The following result shows that under the existence of the $\tilde{\alpha}$-dual GRF $\tilde{X}_{\tilde{\alpha}}$ of $\mathscr{X}_{\alpha}$, with $\tilde{\mathscr{X}}_{\tilde{\alpha}}$ defined on $V_{\tilde{\alpha}},(3.10)$ has a unique solution in the RKHS of $\tilde{\mathscr{X}}_{\tilde{\alpha}}$ which solves the estimation problem in a fractional generalised framework.

THEOREM 3.1. Let $\mathscr{X}_{\alpha}$ be a zero-mean fractional linear functional of the information random field $\mathscr{X}$ with generalised covariance function $B_{\mathscr{X}_{\alpha}}(\cdot, \cdot)$. Assume that $\mathscr{X}_{\alpha}$ has minimum fractional duality order $\tilde{\alpha}$. Then, the estimation problem has a unique solution in the RKHS of the $\tilde{\alpha}$-dual $\tilde{\mathscr{X}}_{\tilde{\alpha}}$ defined by

$$
K^{\prime}(\phi) \underset{\bar{\vartheta}_{\tilde{\alpha}}}{=} R_{\overline{\mathscr{X}}_{\dot{\alpha}}} R_{\mathscr{X}_{\tilde{\alpha}} \mathscr{S}_{\bar{\gamma}}}\left(A^{\prime} \phi\right), \quad \forall \phi \in U_{\beta},
$$


where $R_{\tilde{\mathscr{X}}_{\tilde{\alpha}}}$ is the covariance operator of $\tilde{\mathscr{X}}_{\tilde{\alpha}}$, defined from $V_{\tilde{\alpha}}$ into $U_{\tilde{\alpha}}$, and $R_{\mathscr{X}_{\tilde{\alpha}} \mathscr{S}_{\gamma}}$ is the covariance operator associated with the cross-covariance function $B_{\mathscr{x}_{\tilde{\alpha}} \mathscr{S}_{\gamma}}$ between $\mathscr{X}_{\tilde{\alpha}}$ and $\mathscr{S}_{\gamma}$, defined from $U_{\gamma}$ into $V_{\tilde{\alpha}}$. Moreover,

$$
K(\varphi) \underset{\overline{U_{\beta}}}{=} A R_{\mathscr{S}_{\gamma}, \mathscr{X}_{\tilde{\alpha}}} R_{\tilde{\mathscr{X}}_{\tilde{\alpha}}}(\varphi), \quad \forall \varphi \in V_{\tilde{\alpha}}
$$

which provides a weak-sense solution to the corresponding ordinary estimation problem.

PROOF. From the covariance factorisation of $\mathscr{X}_{\tilde{\alpha}}$ and $\tilde{\mathscr{X}}_{\tilde{\alpha}}$ (see Theorem 2.2), we obtain

$$
\tilde{\mathscr{X}}_{\tilde{\alpha}}\left(R_{\tilde{\mathscr{X}}_{\tilde{\alpha}}}^{-1} \varphi\right)=\tilde{\mathscr{X}}_{\tilde{\alpha}}\left[\left(S_{\tilde{\alpha}}\left(S_{\tilde{\alpha}}^{\prime}\right)^{-1}\right)(\varphi)\right]=\left(\tilde{\mathscr{X}}_{\tilde{\alpha}} S_{\tilde{\alpha}}\right)\left[\left(S_{\tilde{\alpha}}^{\prime}\right)^{-1}(\varphi)\right]=\mathscr{X}_{\tilde{\alpha}}(\varphi)
$$

for all $\varphi \in U_{\tilde{\alpha}}$. Similarly,

$$
\mathscr{X}_{\bar{\alpha}}\left(R_{\tilde{X}_{\tilde{\alpha}}} \phi\right)=\tilde{\mathscr{X}}_{\tilde{\alpha}}(\phi)
$$

for all $\phi \in\left[U_{\tilde{\alpha}}\right]^{*}$. From the duality between $\mathscr{X}_{\tilde{\alpha}}$ and $\tilde{\mathscr{X}}_{\tilde{\alpha}}$ and (3.17), we get, for each $\varphi \in U_{\bar{\alpha}}$, that

$$
\begin{aligned}
0 & =\left\langle\mathscr{X}_{\tilde{\alpha}}(\varphi)-\tilde{\mathscr{X}}_{\tilde{\alpha}}\left(R_{\tilde{\mathscr{X}}_{\tilde{\dot{\alpha}}}^{-1}}^{-1} \varphi\right), \mathscr{X}_{\tilde{\alpha}}(\phi)\right\rangle_{H\left(\mathscr{X}_{\tilde{\alpha})}\right)} \\
& =\left\langle\left(R_{\mathscr{X}_{\tilde{\alpha}}} \varphi\right)^{*}, \phi\right\rangle_{U_{\tilde{\alpha}}}-\left\langle\left(R_{\tilde{\mathscr{X}}_{\tilde{\alpha}}}^{-1} \varphi\right)^{*}, \phi\right\rangle_{U_{\tilde{\alpha}}}=\left\langle\left(R_{\mathscr{X}_{\dot{\alpha}}} \varphi-R_{\tilde{X}_{\tilde{\alpha}}}^{-1} \varphi\right)^{*}, \phi\right\rangle_{U_{\tilde{\alpha}}}
\end{aligned}
$$

for all $\phi \in U_{\tilde{\alpha}}$. Thus

Similarly,

$$
R_{\mathscr{X}_{\tilde{\alpha}}} \varphi=R_{\tilde{\mathscr{X}}_{\tilde{\alpha}}}^{-1} \varphi, \quad \forall \varphi \in U_{\tilde{\alpha}}
$$

$$
R_{\overline{\mathscr{X}}_{\bar{\alpha}} \phi} \phi R_{\mathscr{X}_{\dot{\alpha}}}^{-1} \phi, \quad \forall \phi \in\left[U_{\tilde{\alpha}}\right]^{*} .
$$

These equations give that $R_{\overline{\mathscr{X}}_{\hat{\alpha}}} R_{\mathscr{X}_{\tilde{\alpha}}}$ and $R_{\mathscr{X}_{\tilde{\alpha}}} R_{\tilde{\mathscr{X}}_{\tilde{\alpha}}}$ are the identity operators on $U_{\tilde{\alpha}}$ and $\left[U_{\tilde{\alpha}}\right]^{*}$ respectively. In other words, the existence of the $\tilde{\alpha}$-dual GRF $\tilde{\mathscr{X}}_{\tilde{\alpha}}$ of $\mathscr{X}_{\alpha}$ implies the existence of the inverse covariance operator $R_{\mathscr{X}_{\tilde{\alpha}}}^{-1}$ of the restriction to $U_{\bar{\alpha}}$ of $R_{\mathscr{X}_{\alpha}}$ and $R_{\mathscr{X}_{\dot{\alpha}}}^{-1}=R_{\tilde{\mathscr{x}}_{\tilde{\dot{\alpha}}}}$. Therefore, for each $\phi \in U_{\beta}$, (3.15) provides a solution to (3.10) in the RKHS of $\tilde{\mathscr{X}}_{\bar{\alpha}}, \mathscr{H}\left(\tilde{\mathscr{X}}_{\bar{\alpha}}\right)$.

Similarly, (3.16) provides a solution to (3.13). Since $k(\cdot, \cdot) \in V_{\beta} \otimes U_{\tilde{\alpha}}$, hence, for each $\mathbf{z} \in T, k(\mathbf{z}, \cdot) \in U_{\tilde{\alpha}}$. In the ordinary case, $\tilde{\alpha} \leq 0$ (see Remark 2.3); in particular, in the case where $\tilde{\alpha}<0, k(\mathbf{z}, \cdot)=k_{\mathbf{z}}(\cdot)$ is a distribution, with positive singularity order $\tilde{\alpha}$. Therefore, $k_{\mathbf{z}}(\cdot)$ must be defined in the weak sense. Moreover, in the case where $[-\beta]^{-}>d / 2$, a weak-sense definition of $k_{\mathbf{z}}(\cdot)$ on $V_{\tilde{\alpha}}$ can be obtained as follows:

$$
K(\varphi)(\mathbf{z})=\int_{T} k_{\mathbf{z}}(\mathbf{y}) \varphi(\mathbf{y}) d \mathbf{y}=k_{\mathbf{z}}(\varphi)=A R_{\mathscr{S}_{r} \mathscr{x}_{\dot{\alpha}}} R_{\tilde{\mathscr{x}}_{\dot{\alpha}}}(\varphi)(\mathbf{z}), \quad \forall \varphi \in V_{\tilde{\alpha}}
$$


Indeed, from Sobolev's Embedding Theorem, the weak-sense equality in (3.16) with respect to the $U_{\beta}$-topology becomes a strong-sense equality in view of the continuity of the functions belonging to the space $V_{\beta}$.

\section{Random fields with fractional covariance spectrum}

In the previous section, the minimum fractional duality order $\tilde{\alpha}$ of the information random field $\mathscr{X}_{\alpha}$, which defines the fractional Sobolev space where this covariance operator is an isomorphism, determines the minimum fractional singularity order of the fractional weak-sense solution $K^{\prime}$. Furthermore, the duality order $\tilde{\alpha}$ also specifies the functional space $U_{\tilde{\alpha}}$ where the covariance operator of the information random field is factorised. Thus, the duality condition identifies the second-order properties of the class of random fields for which the estimation problem is solved. In this section we describe such properties in terms of the associated covariance spectrum and RKHS.

From Proposition 2.1, the operators $L=S_{\tilde{\alpha}}^{\prime} \mathscr{I}$ and $\tilde{L}=S_{\tilde{\alpha}} \mathscr{I} I_{\left[U_{\tilde{a}}\right]^{*}}$ are isomorphisms on $U_{\tilde{\alpha}}$ and $\left[U_{\tilde{\alpha}}\right]^{*}$, respectively. Therefore, the operators $\mathscr{I}_{\tilde{\alpha}} L^{-1}: U_{\tilde{\alpha}} \rightarrow L^{2}(T)$ and $\mathscr{I}_{-\tilde{\alpha}} \tilde{L}^{-1}: V_{\tilde{\alpha}} \rightarrow L^{2}(T)$, respectively associated with $R_{\mathscr{X}_{\tilde{\alpha}}}$ (see (2.24)) and $R_{\overline{\mathscr{x}}_{\tilde{\alpha}}}$ (see (2.25)), are bicontinuous. Then, there exist positive constants $C_{1}, C_{2}, \tilde{C}_{1}$, and $\tilde{C}_{2}$ such that

$$
\begin{array}{ll}
C_{1}\|\phi\|_{U_{\tilde{\alpha}}} \leq\left\|\mathscr{I}_{\tilde{\alpha}} L^{-1}(\phi)\right\|_{L^{2}(T)} \leq C_{2}\|\phi\|_{U_{\tilde{\tilde{\alpha}}}}, & \forall \phi \in U_{\tilde{\alpha}}, \\
\tilde{C}_{1}\|\varphi\|_{V_{\tilde{\alpha}}} \leq\left\|\mathscr{I}_{-\tilde{\alpha}} \tilde{L}^{-1}(\varphi)\right\|_{L^{2}(T)} \leq \tilde{C}_{2}\|\varphi\|_{V_{\tilde{\alpha}}}, & \forall \varphi \in V_{\tilde{\alpha}}
\end{array}
$$

These equations are next expressed in terms of the spectra of $R_{\mathscr{x}_{\tilde{\alpha}}}$ and $R_{\overline{\mathscr{x}}_{\hat{\alpha}}}$, respectively.

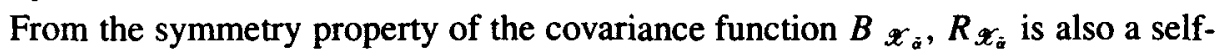
adjoint operator in $L^{2}(T)$. Then, we can consider its representation in terms of a spectral family $\left\{E_{\lambda}\right\}_{\lambda \in \Lambda}$ in $L^{2}(T)$, with $\Lambda$ being the spectrum of $R_{\mathscr{X}_{\dot{\alpha}}}$ (see Section 2):

$$
\left(R_{\mathscr{X}_{\tilde{\alpha}}} \varphi, \psi\right)=\int_{\Lambda} \lambda d\left(E_{\lambda} \varphi, \psi\right)=\int_{\Lambda} \lambda \tilde{\varphi}(\lambda) \tilde{\psi}^{*}(\lambda) d \rho(\lambda), \quad \forall \varphi, \psi \in U_{\tilde{\alpha}},
$$

where $\rho(\cdot)$ denotes the spectral measure of $R_{\mathscr{X}_{\tilde{\boldsymbol{a}}}}$ and

$$
\tilde{\varphi}(\lambda) \tilde{\psi}^{*}(\lambda)=\sum_{j=1}^{N_{\lambda}}\left[\int_{T} \varphi(\mathbf{z}) \phi_{j}^{*}(\mathbf{z}, \lambda) d \mathbf{z}\right]\left[\int_{T} \psi^{*}(\mathbf{y}) \phi_{j}(\mathbf{y}, \lambda) d \mathbf{y}\right] .
$$

Here, $j=1, \ldots, N_{\lambda} \leq \infty$ represent the indexes of the eigenvalues $\lambda_{j} \leq \lambda$ of $R_{X_{\tilde{a}}}$, * means the duality between the fractional Sobolev spaces $U_{\tilde{\alpha}}$ and $V_{\tilde{\alpha}}$ with respect to $L^{2}(T)$, and, for each $\lambda \in \Lambda,\left\{\phi_{j}(\cdot, \lambda)\right\}_{j \leq N(\lambda)} \subseteq U_{\bar{\alpha}}$ and $\left\{\phi_{j}^{*}(\cdot, \lambda)\right\}_{j \leq N(\lambda)} \subseteq V_{\tilde{\alpha}}$ are dual 
Riesz bases with respect to $L^{2}(T)$. Such bases provide the following representation of the spectral kernel $\Phi(\cdot, \cdot, \lambda)$ of $R_{\mathscr{X}_{\tilde{\alpha}}}$ :

$$
\Phi(\mathbf{z}, \mathbf{y}, \lambda)=\sum_{j=1}^{N_{\lambda}} \phi_{j}(\mathbf{z}, \lambda) \phi_{j}^{*}(\mathbf{y}, \lambda)
$$

(see Ramm [21, pages 145-147]). Note that (4.4) can also be interpreted as the inner product in $L^{2}(T)$ of the functions

$$
\begin{aligned}
\tilde{\varphi}(\lambda)(\mathbf{y}) & =\sum_{j=1}^{N_{\mathbf{2}}}\left[\int_{T} \varphi(\mathbf{z}) \phi_{j}^{*}(\mathbf{z}, \lambda) d \mathbf{z}\right] \phi_{j}(\mathbf{y}, \lambda), \quad \text { and } \\
\tilde{\psi}^{*}(\lambda)(\mathbf{y}) & =\sum_{j=1}^{N_{\mathbf{\lambda}}}\left[\int_{T} \psi^{*}(\mathbf{z}) \phi_{j}(\mathbf{z}, \lambda) d \mathbf{z}\right] \phi_{j}^{*}(\mathbf{y}, \lambda) .
\end{aligned}
$$

In addition, from Proposition 2.1 and Theorem 2.2, $R_{\tilde{\mathscr{X}}_{\tilde{a}}}$ is a bounded function of $R_{\mathscr{X}_{\dot{\alpha}}}$. Hence, it can also be represented in terms of $\Phi$ and $\rho$ as

$$
\left(R_{\tilde{\mathscr{X}}_{\tilde{\bar{\alpha}}}}(\phi), \psi\right)=\int_{\Lambda} \lambda^{-1} \tilde{\phi}(\lambda) \tilde{\psi}(\lambda) d \rho(\lambda), \quad \forall \phi, \psi \in V_{\tilde{\alpha}}
$$

From (4.3) and (4.6), equations (4.1) and (4.2) can be respectively written as

$$
\begin{aligned}
C_{1} \int_{\mathbb{R}^{n}}\left(1+|\lambda|^{2}\right)^{\bar{\alpha}}|\hat{\phi}(\lambda)|^{2} d \lambda & \leq \int_{\Lambda}|\lambda||\tilde{\phi}(\lambda)|^{2} d \rho(\lambda) \\
& \leq C_{2} \int_{\mathbb{R}^{n}}\left(1+|\lambda|^{2}\right)^{\tilde{\alpha}}|\hat{\phi}(\lambda)|^{2} d \lambda, \\
\tilde{C}_{1} \int_{\mathbb{R}^{n}}\left(1+|\lambda|^{2}\right)^{-\tilde{\alpha}}|\hat{\varphi}(\lambda)|^{2} d \lambda & \leq \int_{\Lambda}|\lambda|^{-1}|\tilde{\varphi}(\lambda)|^{2} d \rho(\lambda) \\
& \leq \tilde{C}_{2} \int_{\mathbb{R}^{n}}\left(1+|\lambda|^{2}\right)^{-\tilde{\alpha}}|\hat{\varphi}(\lambda)|^{2} d \lambda,
\end{aligned}
$$

for $\phi \in U_{\bar{\alpha}}$ and $\varphi \in V_{\tilde{\alpha}}$, where $\hat{\phi}$ and $\hat{\varphi}$ stand for the Fourier transforms of $\phi$ and $\varphi$, respectively, and, where for each $\lambda \in \Lambda,|\tilde{\phi}|^{2}=\tilde{\phi} \tilde{\phi}^{*}$ and $|\tilde{\varphi}|^{2}=\tilde{\varphi} \tilde{\varphi}^{*}$ are defined as in (4.4).

As a consequence, we have the following result:

THEOREM 4.1. Under conditions of Theorem 3.1, the following assertions hold:

(i) $\phi \in U_{\tilde{\alpha}}$ if and only if $\left(R_{\mathscr{X}_{\tilde{\alpha}}} \phi, \phi\right)<\infty$, that is, if and only if $\phi \in \mathscr{H}\left(\tilde{\mathscr{X}}_{\tilde{\alpha}}\right)$.

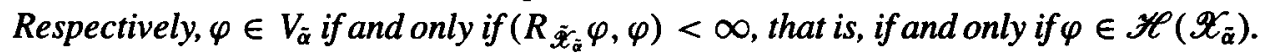


(ii) In the case where $\mathscr{L}_{\tilde{\alpha}}=\mathscr{I}_{\tilde{\alpha}} L^{-1}$ and $\mathscr{L}_{\tilde{\alpha}}^{-1}=\left[\mathscr{I}_{-\tilde{\alpha}} \tilde{L}^{-1}\right]^{\prime}$ are self-adjoint, the following equivalences hold:

$$
\begin{gathered}
\varphi \in \bar{H}^{\tilde{\alpha}}(T)=U_{\tilde{\alpha}} \Leftrightarrow \int_{\Lambda}\left(1+|\lambda|^{2}\right)|\tilde{\varphi}(\lambda)|^{2} d \rho_{\mathscr{L}_{\tilde{\alpha}}}<\infty, \\
\psi \in H^{-\tilde{\alpha}}(T)=V_{\tilde{\alpha}} \Leftrightarrow \int_{\Lambda}\left(1+|\lambda|^{2}\right)|\tilde{\psi}(\lambda)|^{2} d \rho_{\left[\mathscr{L}_{\tilde{\sigma}}^{-1}\right]^{\prime}}(\lambda)<\infty .
\end{gathered}
$$

(iii) In addition, in the case where $\mathscr{L}_{\bar{\alpha}}$ commutes with the Bessel potential of order $\tilde{\alpha}$, we obtain

$$
\varphi \in \bar{H}^{\beta \tilde{\alpha}}(T) \Leftrightarrow \int_{\Lambda}\left(1+|\lambda|^{2}\right)^{\beta}|\tilde{\varphi}(\lambda)|^{2} d \rho_{\mathscr{L}_{\dot{\boldsymbol{q}}}}<\infty
$$

PROOF. (i) From (4.7) and (4.8),

$$
\phi \in \bar{H}^{\check{\alpha}}(T) \Leftrightarrow\left(R_{X_{\tilde{\alpha}}} \phi, \phi\right)<\infty, \quad \varphi \in H^{\tilde{\alpha}}(T) \Leftrightarrow\left(R_{\tilde{X}_{\dot{\alpha}}} \varphi, \varphi\right)<\infty .
$$

The second part of (i) is derived from Proposition 2.1 (i), since

$$
\left(R_{X_{\tilde{\alpha}}}(\phi), \phi\right)=B_{X_{\dot{\alpha}}}(\phi, \phi)=\left\langle X_{\tilde{\alpha}}(\phi), X_{\tilde{\alpha}}(\phi)\right\rangle_{H\left(X_{\dot{\alpha}}\right)}=\langle\phi, \phi\rangle_{\mathscr{H}\left(\tilde{X}_{\dot{\alpha}}\right)}=\|\phi\|_{\mathscr{H}\left(\tilde{X}_{\dot{\alpha}}\right)}^{2},
$$

for all $\phi \in U_{\tilde{\alpha}}$, and

$$
\left(R_{\tilde{X}_{\tilde{a}}} \varphi, \varphi\right)=\|\varphi\|_{\mathscr{H}\left(\mathscr{X}_{\tilde{\mathbf{x}}}\right)}^{2},
$$

for all $\varphi \in V_{\tilde{\alpha}}$.

(ii) As $\mathscr{L}_{\tilde{\alpha}}$ and $\left[\mathscr{L}_{\tilde{\alpha}}^{-1}\right]^{\prime}=\mathscr{L}_{\bar{\alpha}}^{-1}$ are bicontinuous operators from $U_{\tilde{\alpha}}$ into $L^{2}(T)$ and from $V_{\hat{\alpha}}$ into $L^{2}(T)$, respectively, the following inequalities hold:

$$
\begin{aligned}
N_{1} \int_{\mathbb{R}^{n}}\left(1+|\lambda|^{2}\right)^{\tilde{\alpha}}|\hat{\varphi}(\lambda)|^{2} d \lambda & \leq \int_{\Lambda}|\lambda|^{2}|\tilde{\varphi}(\lambda)|^{2} d \rho_{\mathscr{L}_{\dot{\alpha}}}(\lambda) \\
& \leq N_{2} \int_{\mathbb{R}^{n}}\left(1+|\lambda|^{2}\right)^{\tilde{\alpha}}|\hat{\varphi}(\lambda)|^{2} d \lambda, \\
\tilde{N}_{1} \int_{\mathbb{R}^{n}}\left(1+|\lambda|^{2}\right)^{-\alpha}|\hat{\psi}(\lambda)|^{2} d \lambda & \leq \int_{\Lambda}|\lambda|^{-2}|\tilde{\psi}(\lambda)|^{2} d \rho_{\mathscr{L}_{\dot{\alpha}}}(\lambda) \\
& =\int_{\Lambda}|\lambda|^{2}|\tilde{\psi}(\lambda)|^{2} d \rho_{\left[\mathscr{L}_{\dot{\alpha}}^{-1} \gamma\right.} \\
& \leq \tilde{N}_{2} \int_{\mathbb{R}^{n}}\left(1+|\lambda|^{2}\right)^{-\alpha}|\hat{\psi}(\lambda)|^{2} d \lambda,
\end{aligned}
$$

for $\varphi \in \breve{H}^{\tilde{\alpha}}(T)$, and $\psi \in H^{-\tilde{\alpha}}(T)$, with $N_{1}, N_{2}, \tilde{N}_{1}$ and $\tilde{N}_{2}$ being positive constants, and with $\rho_{\mathscr{L}_{\tilde{\alpha}}}$ and $\rho_{\left[\mathscr{L}_{\dot{\alpha}}^{-1}\right\}^{\prime}}$ being the spectral measures associated with $\mathscr{L}_{\tilde{\alpha}}$ and $\left[\mathscr{L}_{\tilde{\alpha}}^{-1}\right]^{\prime}$, respectively. The assertion (ii) is straightforward from (4.14) and (4.15).

(iii) The right hand side of (4.11) is finite if and only if $\varphi$ belongs to the domain of $\mathscr{L}_{\bar{\alpha}}^{\beta}$; in the case where $\mathscr{L}_{\tilde{\alpha}}$ commutes with the Bessel potential of order $\tilde{\alpha}$, this is equivalent to $\varphi \in \bar{H}^{\bar{\alpha} \beta}(T)$. 
Note that the assumption in (iii) is not equivalent to the assumption that $\mathscr{L}_{\tilde{\alpha}}$ be a function of the Bessel potential of order $\tilde{\alpha}$ (see Dautray and Lions [11, page 145]).

In the following corollary we specify an important class of random fields for which the estimation problem is solved by applying Theorem 3.1.

COROLLARY 4.2. For each $\beta \in \mathbb{Q}$, we consider the set $\mathscr{F}_{\beta}$ of real-valued, positive and continuous functions $F_{\beta}$ satisfying

$$
C \leq F_{\beta}(\lambda)\left(1+|\lambda|^{2}\right)^{\beta / 2} \leq C^{\prime}, \quad C, C^{\prime}>0,
$$

and define the following class $\mathscr{R}_{\beta}, \beta \in \mathbb{Q}$, of covariance operators:

$$
R_{\beta} \in \mathscr{R}_{\beta} \quad \Leftrightarrow \quad R_{\beta}=F_{\beta}\left(\mathscr{L}_{\tilde{\alpha}}\right), \text { for some } F_{\beta} \in \mathscr{F}_{\beta},
$$

where $\mathscr{L}_{\tilde{\alpha}}$ is a self-adjoint elliptic fractional differential operator of order $\tilde{\alpha}$ on $L^{2}(T)$. Then the estimation problem is solved as in Theorem 3.1 for this class of information random fields.

PROOF. If $\mathscr{L}_{\tilde{\alpha}}$ is a self-adjoint elliptic fractional differential operator of order $\tilde{\alpha}$ on $L^{2}(T)$, then $\mathscr{L}_{\tilde{\alpha}}$ satisfies condition (4.11). Clearly, $R_{\beta}$ is an isomorphism from $V_{\beta \tilde{\alpha} / 2}$ into $U_{\beta \tilde{\alpha} / 2}$. Thus, if $\mathscr{X}_{\beta \tilde{\alpha} / 2}$ represents a fractional generalised information random field with covariance operator $R_{\beta}$ satisfying condition (4.17), then its $\beta \tilde{\alpha} / 2$-dual $\tilde{\mathscr{X}}_{\beta \tilde{\alpha} / 2}$ exists. Therefore, Theorem 3.1 provides a solution to the estimation problem in a fractional generalised framework for this class of information random fields.

In particular, the estimation problem is solved in Ramm [21] for the case where $\mathscr{L}_{\tilde{\alpha}}$ is a self-adjoint elliptic differential operator of integer order on $L^{2}(T)$, and

$$
F_{\beta}(\lambda)=\frac{P(\lambda)}{Q(\lambda)}
$$

with $P(\lambda)$ and $Q(\lambda)$ being positive polynomials of respective orders $p$ and $q$ satisfying

$$
\begin{aligned}
& 0<c_{1} \leq P(\lambda)\left(1+|\lambda|^{2}\right)^{-p / 2} \leq c_{2}, \\
& 0<c_{3} \leq Q(\lambda)\left(1+|\lambda|^{2}\right)^{-q / 2} \leq c_{4},
\end{aligned}
$$

for some positive constants $c_{1}, c_{2}, c_{3}$ and $c_{4}$. That is, $\beta=q-p$, and $\tilde{\alpha} \in \mathbb{N}$.

\section{Final comments}

In this paper we have considered the problem of estimating on a bounded domain $T \subseteq \mathbb{R}^{d}$ the output random field of a stochastic equation from the information provided 
by the observation of the input random field with additive noise on $T$. In the case where the fractional generalised information random field has minimum fractional duality order $\tilde{\alpha}$, the information used in the estimation is provided by the test functions in the space $U_{\tilde{\alpha}}$. Furthermore, if the sample information is provided by a closed subset $U_{\alpha}^{I}$ of test functions in $U_{\alpha}$, then the useful information for the estimation is given in terms of the test functions in $U_{\tilde{\alpha}}^{I}=\mathscr{I}_{-(\tilde{\alpha}-\alpha)}\left(U_{\alpha}^{I}\right)$. The weak-sense solution to the estimation problem is then derived in a similar way to the case considered in this paper by defining $K^{\prime}$ as a linear operator from $U_{\beta}$ into $U_{\tilde{\alpha}}^{l}$. In particular, the situation where the ordinary information random field is observed in a subdomain $T^{\prime} \subseteq T$ is included in the above mentioned case with $U_{\tilde{\alpha}}^{l}=\bar{H}^{\tilde{\alpha}}\left(T^{\prime}\right)$. Finally, we note that if the interest is in approximating the fractional generalised output random field in a subspace $U$, instead of the whole space $U_{\beta}$, the estimation function $k(\cdot, \cdot)$ will be found in a wider class, with operator $K^{\prime}$ being defined from $U$ into $U_{\tilde{\alpha}}$, the space of regularisation of the information.

\section{References}

[1] V. V. Anh, J. M. Angulo and M. D. Ruiz-Medina, 'Possible long-range dependence in fractional random fields', J. Statist. Plan. Inference 80 (1999), 95-110.

[2] V. V. Anh, J. M. Angulo, M. D. Ruiz-Medina and Q. Tieng, 'Long-range dependence and secondorder ontermittency of two-dimensional turbulence', Environ. Modelling and Software 13 (1998), 233-238.

[3] V. V. Anh and C. C. Heyde (eds.), 'Special issue on long-range dependence', J. Statist. Plan. Inference 80 (1999).

[4] V. V. Anh, F. Gras and H. T. Tsui, 'Multifractal description of natural scenes', Fractals 4 (1996), 35-43.

[5] V. V. Anh, M. D. Ruiz-Medina and J. M. Angulo, 'Covariance factorisation and abstract representation of generalised random fields', Bull. Austral. Math. Soc. 62 (2000), 319-334.

[6] A. L. Barabasi and H. E. Stanley, Fractal concepts in surface growth (Cambridge University Press, London, 1995).

[7] M. F. Barnsley and L. P. Hurd, Fractal image compression (A. K. Peters, 1993).

[8] J. Beran, Statistics for long-memory processes (Chapman and Hall, New York, 1994).

[9] I. N. Bronshtein and K. A. Semendyayer, Handbook of mathematics (Verlag Harri Deutsch, Van Nostrand Reinhold Company, New York, 1985).

[10] R. Dautray and J. L. Lions, Mathematical analysis and numerical methods for science and technology, Vol. 2, Functional and variational methods (Springer, New York, 1985).

[11] - Mathematical analysis and numerical methods for science and technology, Vol. 3, Spectral theory and applications (Springer, New York, 1985).

[12] K. Falconer, Techniques in fractal geometry (Wiley, New York, 1997).

[13] M. Farge, J. Hunt. and C. Vassilicos (eds.), Wavelets, fractals and Fourier transform: new developments and new applications (Clarendon, New York, 1993).

[14] U. Frisch, Turbulence (Cambridge University Press, London, 1995).

[15] I. M. Gel'fand and N. Y. Vilenkin, Generalized functions, Vol. 4 (Academic Press, New York, 1964). 
[16] P. M. Innacone and M. Khokha (eds.), Fractal geometry in biological systems (CRC Press, 1996).

[17] T. A. Kailath, 'A view of three decades of linear filtering theory', IEEE Trans. Inform. Theory IT-20 (1974), 145-181.

[18] B. B. Mandelbrot and J. W. Van Ness, 'Fractional Brownian motion, fractional noises and applications', SIAM Review 10 (1968), 422-437.

[19] E. Peters, Fractal market analysis (Wiley, New York, 1994).

[20] A. G. Ramm, Theory and applications of some new classes of integral equations (Springer, New York, 1980).

[21] - Random fields estimation theory (Longman, Essex, 1990).

[22] Y. A. Rozanov, Markov random fields (Springer, New York, 1982).

[23] M. D. Ruiz-Medina, J. M. Angulo and V. V. Anh, 'Fractional generalised random fields', submitted.

[24] L. Schwartz, Théorie des Distributions (Hermann, Paris, 1966).

[25] E. M. Stein, Singular integrals and differential properties of functions (Princeton University Press, New Jersey, 1970).

[26] H. Triebel, Interpolation theory, function spaces, differential operators (North-Holland, New York, 1978).

Departamento de Estadística

e Investigación Operativa

Universidad de Granada

Campus Fuente Nueva s/n

E-18071 Granada

Spain

e-mail: jmangulo@goliat.ugr.es
School of Mathematical Sciences Queensland University of Technology GPO Box 2434 Brisbane QLD 4001 Australia e-mail: v.anh@fsc.qut.edu.au 